Published in final edited form as:

Nat Genet. 2019 May ; 51(5): 793-803. doi:10.1038/s41588-019-0397-8.

Users may view, print, copy, and download text and data-mine the content in such documents, for the purposes of academic research, subject always to the full Conditions of use:http://www.nature.com/authors/editorial_policies/license.html\#terms

${ }^{\&}$ Correspondence to: jkelsoe@ucsd.edu or eli.stahl@mssm.edu.

$\dagger$ Equal contribution

*Co-last authors

Author Contributions:

Writing group: E.A.S., G.B., A.J.F., A.McQuillin, S.R., J.R.I.C., N.M., N.R.W., A.D.F., H.J.E., S.C., R.A.O., L.J.S., O.A.A., J.K. PGC BD PI group: E.A.S., G.B., A.J.F., A.McQuillin, D.Curtis, R.H.P., R.A., I.A., M.A., L.B., B.T.B., F.B., W.H.B., J.M.B., D.H.R.B., M.Boehnke, A.D.B., A.C., N.C., U.D., T.Esko, B.E., M.Frye, J.M.F., E.S.G., M.G., F.G., M.G-S., J.H., D.M.H., C.M.H., I.J., L.A.J., R.S.K., M.Landén, M.Leboyer, C.M.L., Q.S.L., J.Lissowska, N.G.M., S.L.M., A.M.M., F.J.M., I.M., A.Metspalu, P.B.Mitchell, G.M., O.M., P.B.Mortensen, B.M-M., R.M.M., B.M.N., V.N., M.N., M.M.N., M.C.O’D., K.J.O., M.J.O., S.A.P., C.P., M.T.P., J.A.R-Q., M.Ribasés, M.Rietschel, G.A.R., M.Schalling, P.R.S., T.G.S., A.S., J.W.S., H.S., K.S., E.Stordal, G.T., A.E.V., E.V., J.B.V., T.W., J.I.N., A.D.F., H.J.E., S.C., R.A.O., L.J.S., O.A.A., J.K., P.S

Bioinformatics: E.A.S., G.B., A.J.F., J.R.I.C., H.A.G., P.A.H., S.E.B., D.W.C., V.E-P., C.G., M.L.H., S.H., R.Karlsson, M.Leber, C.Liu, F.Meng, L.M.O.L., A.P.S.O., C.S.R., P.R., P.D.S., M.Steffens, S.Szelinger, T.E.T., S.X., P.Zandi, eQTLGen Consortium, BIOS Consortium, T.Esko, E.S.G., Q.S.L., G.A.R., H.S.

Clinical: A.McQuillin, M.M., E.A., N.A-R., A.A., N.B., M.Bauer, C.B.P., E.B., M.P.B., M.Budde, M.Burmeister, W.Byerley, M.C., P.C., W.C., D.Curtis, P.M.C., J.R.D., T.Elvsåshagen, L.Forty, C.F., K.G., J.Garnham, M.G.P., K.G-S., M.J.G., J.Grove, J.G-P., M.Hautzinger, U.H., M.Hipolito, A.J., J.L.K., S.K-S., M.K., R.Kupka, C.Lavebratt, J.Lawrence, W.B.L., S.L., D.J.M., P.B.Mahon, W.M., L.Martinsson, P.M., M.G.M., H.M., A.N.A., E.A.N., C.O’D., L.O., U.Ö., R.H.P., A.Perry, A.Pfennig, J.B.P., E.J.R., A.R., J.P.R., F.R., M.Rivera, W.A.S., C.S.W., E.Sigurdsson, C.S., O.B.S., J.L.S., A.T.S., D.S.C., J.S.S., F.S., J.S., R.C.T., H.V., T.W.W., A.H.Y., R.A., I.A., M.A., B.T.B., F.B., D.H.R.B., A.D.B., A.C., N.C., U.D., B.E., M.Frye, E.S.G., M.G., F.G., M.G-S., J.H., I.J., L.A.J., R.S.K., G.K., M.Landén, J.Lissowska, N.G.M., F.Mayoral, S.L.M., A.M.M., F.J.M., I.M., P.B.Mitchell, G.M., O.M., P.B.Mortensen, V.N., M.C.O’D., K.J.O., M.J.O., C.P., M.T.P., J.A.R-Q., M.Rietschel, T.G.S., A.S., J.W.S., E.Stordal, A.E.V., E.V., J.I.N., A.D.F.

Genomic assays/data generation: G.B., A.J.F., E.A., D.A., M.B-H., C.B.P., J.B-G., T-K.C., D.W.C., C.Cruceanu, F.D., J.D-F., S.D., S.B.F., J.F., M.G.P., E.K.G., P.H., S.J., R.Kandaswamy, A.C.K., S.E.L., A.Maaser, J.D.M., L.Milani, G.W.M., D.W.M., T.W.M., E.R., C.S-M., T.S., C.S.H., S.Szelinger, J.T., S.H.W., P.Zhang, A.C., T.Esko, J.M.F., E.S.G., M.G., D.M.H., R.M.M., M.M.N., M.Ribasés, G.A.R., G.T., S.C.

Obtained funding for BD samples: G.B., H.A., N.A-R., J.D.B., W.Bunney, J.R.D., N.B.F., L.Frisén, M.J.G., S.J., J.A.K., C.Lavebratt, S.L., P.M., G.W.M., U.Ö., J.B.P., M.Rivera, A.F.S., C.S.W., S.J.W., T.W.W., A.H.Y., M.A., B.T.B., W.H.B., J.M.B., M.Boehnke, A.D.B., A.C., N.C., M.Frye, J.M.F., E.S.G., M.G., M.G-S., I.J., L.A.J., M.Landén, M.Leboyer, C.M.L., N.G.M., A.Metspalu, P.B.Mitchell, O.M., P.B.Mortensen, B.M.N., M.N., M.M.N., M.C.O’D., M.J.O., C.P., M.T.P., M.Rietschel, G.A.R., P.R.S., T.G.S., J.W.S., G.T., J.B.V., T.W., J.I.N., H.J.E., R.A.O., P.S.

Statistical analysis: E.A.S., G.B., S.R., V.T., M.M., Y.W., J.R.I.C., H.A.G., C.A.d.L., S.Steinberg, J.M.W.P., M.T., E.M.B., T.H.P., P.A.H., A.L.R., L.A., N.A-R., T.D.A., V.A., S.A., J.A.B., R.B., S.E.B., J.B., F.C., K.C., A.W.C., D.Chen, C.Churchhouse, A.M.D., S.d.J., A.L.D., A.D., V.E-P., C.C.F., M.Flickinger, T.M.F., D.G., C.G., J.Goldstein, S.D.G., T.A.G., J.Grove, W.G., M.L.H., D.H., L.H., J.S.J., R.Karlsson, M.Leber, P.H.L., J.Z.L., S.M., S.E.M., D.W.M., N.M., H.N., C.M.N., L.M.O.L., A.P.S.O., S.M.P., C.S.R., P.R., D.M.R., N.J.S., O.B.S., T.E.T., W.W., W.X., P.Zandi, P.Zhang, S.Z., eQTLGen Consortium, BIOS Consortium, J.M.B., A.D.B., M.J.D., E.S.G., F.G., Q.S.L., B.M-M., D.P., H.S., P.F.S., N.R.W., P.S.

Competing Interest:

T.E. Thorgeirsson, S. Steinberg, H. Stefansson and K. Stefansson are employed by deCODE Genetics/Amgen. Multiple additional authors work for pharmaceutical or biotechnology companies in a manner directly analogous to academic co-authors and collaborators. A.H. Young has given paid lectures and is on advisory boards for the following companies with drugs used in affective and related disorders: Astra Zeneca, Eli Lilly, Janssen, Lundbeck, Sunovion, Servier, Livanova. A.H. Young is Lead Investigator for Embolden Study (Astra Zeneca), BCI Neuroplasticity study and Aripiprazole Mania Study, which are investigator-initiated studies from Astra Zeneca, Eli Lilly, Lundbeck, and Wyeth. J. Nurnberger is an investigator for Janssen. P.F. Sullivan reports the following potentially competing financial interests: Lundbeck (advisory committee), Pfizer (Scientific Advisory Board member), and Roche (grant recipient, speaker reimbursement). G. Breen reports consultancy and speaker fees from Eli Lilly and Illumina and grant funding from Eli Lilly. O.A. Andreassen has received speaker fees from Lundbeck. J.Antoni Ramos-Quiroga was on the speakers' bureau and/or acted as consultant for Eli-Lilly, Janssen-Cilag, Novartis, Shire, Lundbeck, Almirall, Braingaze, Sincrolab, and Rubió in the last 5 years. He also received travel awards (air tickets + hotel) for taking part in psychiatric meetings from Janssen-Cilag, Rubió, Shire, and Eli- Lilly. The Department of Psychiatry chaired by him received unrestricted educational and research support from the following companies in the last 5 years: Eli-Lilly, Lundbeck, Janssen- Cilag, Actelion, Shire, Ferrer, Oryzon, Roche, Psious, and Rubió. Dr. E. Vieta has received grants and served as consultant, advisor or CME speaker for the following entities: AB-Biotics, Abbott, Allergan, Angelini, AstraZeneca, Bristol-Myers Squibb, Dainippon Sumitomo Pharma, Farmindustria, Ferrer, Forest Research Institute, Gedeon Richter, Glaxo-Smith-Kline, Janssen, Lundbeck, Otsuka, Pfizer, Roche, SAGE, Sanofi-Aventis, Servier, Shire, Sunovion, Takeda, the Brain and Behaviour Foundation, the Catalan Government (AGAUR and PERIS), the Spanish Ministry of Science, Innovation, and Universities (AES and CIBERSAM), the Seventh European Framework Programme and Horizon 2020, and the Stanley Medical Research Institute. T. Elvsåshagen has received speaker fees from Lundbeck. All other authors declare no financial interests or potential conflicts of interest. 


\title{
Genome-wide association study identifies 30 Loci Associated with Bipolar Disorder
}

\author{
A full list of authors and affiliations appears at the end of the article.
}

\section{Abstract}

Bipolar disorder is a highly heritable psychiatric disorder. We performed a genome-wide association study including 20,352 cases and 31,358 controls of European descent, with follow-up analysis of 822 variants with $\mathrm{P}<1 \times 10^{-4}$ in an additional 9,412 cases and 137,760 controls. Eight of the 19 variants that were genome-wide significant (GWS, $\mathrm{p}<5 \times 10^{-8}$ ) in the discovery GWAS were not GWS in the combined analysis, consistent with small effect sizes and limited power but also with genetic heterogeneity. In the combined analysis 30 loci were GWS including 20 novel loci. The significant loci contain genes encoding ion channels, neurotransmitter transporters and synaptic components. Pathway analysis revealed nine significantly enriched gene-sets including regulation of insulin secretion and endocannabinoid signaling. BDI is strongly genetically correlated with schizophrenia, driven by psychosis, whereas BDII is more strongly correlated with major depressive disorder. These findings address key clinical questions and provide potential new biological mechanisms for BD.

Bipolar disorder (BD) is a severe neuropsychiatric disorder characterized by recurrent episodes of mania and depression that affect thought, perception, emotion, and social behaviour. A lifetime prevalence of 1-2\%, elevated morbidity and mortality, onset in young adulthood, and a frequently chronic course make BD a major public health problem and a leading cause of the global burden of disease ${ }^{1}$. Clinical, twin and molecular genetic data all strongly suggest that $\mathrm{BD}$ is a multifactorial disorder ${ }^{2}$. Based on twin studies, the overall heritability of BD has been estimated to be more than $70 \%^{3,4}$, suggesting a substantial involvement of genetic factors in the development of the disorder, although non-genetic factors also influence risk.

BD can be divided into two main clinical subtypes 5,6 : bipolar I disorder (BD1) and bipolar II disorder (BD2). In BD1, manic episodes typically alternate with depressive episodes during the course of illness. Diagnosis of BD2 is based on the lifetime occurrence of at least one depressive and one hypomanic (but no manic) episode. Although modern diagnostic systems retain the Kraepelinian dichotomy ${ }^{7}$ between BD and schizophrenia (SCZ), the distinction between the two disorders is not always clear-cut, and patients who display clinical features of both disorders may receive a diagnosis of schizoaffective disorder-bipolar type (SAB). Likewise, in genetic studies BD and SCZ are usually treated separately, although recent epidemiological and molecular genetic studies provide strong evidence for some overlap between the genetic contributions to their etiology 2,8 .

Recent genome-wide association studies (GWAS) in BD have identified a number of significant associations between disease status and common genetic variants ${ }^{9-23}$. The first large collaborative BD GWAS by the multinational Psychiatric Genomics Consortium 
(PGC) Bipolar Disorder Working Group comprised 7,481 BD patients and 9,250 controls and identified four genome-wide significant loci ${ }^{9}$. Three subsequent meta-analyses that included the PGC BD data $10,12,18$ identified an additional 5 loci.

Estimates of the proportion of variance in liability attributable to common variants genomewide (SNP-heritability) indicate that $\sim 30 \%$ of the heritability for BD is due to common genetic variants ${ }^{8}$. To date, only a small fraction of this heritability is explained by associated loci, but results from other human complex traits suggest that many more will be identified by increasing the sample size of GWAS ${ }^{24}$. Here, we report the second GWAS of the PGC Bipolar Disorder Working Group, comprising 20,352 cases and 31,358 controls of European descent in a single, systematic analysis, with follow up of top findings in an independent sample of 9,412 cases and 137,760 controls. Some of our findings reinforce specific hypotheses regarding $\mathrm{BD}$ neurobiology; however, the majority of the findings suggest new biological insights.

\section{RESULTS}

\section{GWAS of bipolar disorder (BD)}

We performed a GWAS meta-analysis of 32 cohorts from 14 countries in Europe, North America and Australia (Supplementary Table 1A), totaling 20,352 cases and 31,358 controls of European descent (effective sample size 46,582). This is a large GWAS of BD, a 2.7-fold increase in the number of cases compared to our previous GWAS ${ }^{9}$, and includes 6,328 case and 7,963 control samples not previously reported. We imputed variant dosages using the 1,000 Genomes reference panel, retaining association results for 9,372,253 autosomal variants with imputation quality score INFO $>0.3$ and minor allele frequency $\geq 1 \%$ in both cases and controls. We performed logistic regression of case status on imputed variant dosage using genetic ancestry covariates. The resulting genomic inflation factor $\left(\lambda_{\mathrm{GC}}\right)$ was $1.23,1.01$ when scaled to 1,000 cases and 1,000 controls $\left(\lambda_{1000}\right)$ (Supplementary Figure 1). The LD Score regression intercept was 1.021 ( $\mathrm{se}=0.010$ ), and the attenuation ratio of 0.053 ( $\mathrm{se}=0.027)$ was non-significant, indicating that the observed genomic inflation is indicative of polygenicity rather than stratification or cryptic population structure ${ }^{25}$. The LD-score regression SNP-heritability estimates for BD were $0.17-0.23$ on the liability scale assuming population prevalence of 0.5-2\%. See Supplementary Table 1A, Online Methods and Supplementary Note for sample and method details.

We find a marked increase in phenotypic variance explained by genome-wide polygenic risk scores (PRS) compared to previous publications (sample size weighted mean observed Nagelkerke's $R^{2}=0.08$ across datasets, liability scale $R^{2}=0.04$, for $p$-threshold 0.01 ; Supplementary Figure 2 and Supplementary Table 2). Among the different datasets, we observed no association between the PRS $\mathrm{R}^{2}$ and: (i) the gender distribution of the BD cases ( $\mathrm{p}=0.51$ ); (ii) the proportion of cases with psychosis ( $\mathrm{p}=0.61$ ); (iii) the proportion with a family history of $\mathrm{BD}(\mathrm{p}=0.82)$; or (iv) the median age of onset for $\mathrm{BD}(\mathrm{p}=0.64)$. In our primary genome-wide analysis, we identified 19 loci exceeding genome-wide significance $\left(\mathrm{P}<5 \times 10^{-8}\right.$; Table 1$)$. 


\section{Follow-up of suggestive loci in additional samples}

We tested lead variants that were significant at $\mathrm{P}<1 \times 10^{-4}$ in our discovery GWAS metaanalysis, a total of 794 autosomal and $28 \mathrm{X}$ chromosome variants, for association in followup samples totaling 9,412 cases and 137,760 controls of European ancestry (effective sample size 23,005; Supplementary Note and Supplementary Table 1B). We first compared discovery and follow-up sample summary statistics using LD score regression, and estimated their genetic correlation to be 0.98 ( $\mathrm{se}=0.07$ ), consistent with homogeneous genetic effects between the two samples. Discovery and follow-up samples also show similar patterns of significant genetic correlations with a range of other human diseases and traits in the LD Hub database ${ }^{26}$ (Supplementary Table 3; correlation of $0.93, p=8.3 \times 10^{-14}$, Supplementary Figure 3).

Thirty autosomal loci achieved genome-wide significance $\left(\mathrm{P}<5 \times 10^{-8}\right)$ in fixed-effect metaanalysis of our GWAS and follow-up samples (Figure 1, Table 1A, Supplementary Data 1-3, Supplementary Table 4). In Supplementary Table 5, we present detailed descriptions of the associated loci and genes, with bioinformatic and literature evidence for their potential roles in BD. Of the 30 genome-wide significant loci from our combined analysis, 20 are novel BD risk loci. These include 19 loci that were significant only in the combined analysis, of which three were reported to have genome-wide significant SNPs in previous studies ( $A D C Y 2^{18}$, $P O U 3 F 2^{18}, A N K 3^{12,18}$ ), and 11 that were significant in our primary GWAS. We refer to loci by the gene name attributed in previous BD GWAS publications, or by the name of the closest gene for novel loci, without implication that the named gene is causal. Results for all variants tested in the follow-up study are presented in Supplementary Table 4.

Of the 19 variants that were genome-wide significant in the discovery GWAS, 8 were not genome-wide significant in the combined analysis (Table 1B), and 11 were non-significant in one-tailed association tests in the follow-up samples ( $p>0.05$ in Table 1). Still, the followup results for these 19 variants are clearly non-null in aggregate: all 19 had consistent directions of effect between discovery GWAS and follow-up ( 9.5 expected by chance, binomial test $\mathrm{p}=4 \times 10^{-6}$ ), and eight of the 19 had follow-up 1-tailed $\mathrm{p}<0.05$ ( 1 expected by chance, sign test $\left.\mathrm{p}=2 \times 10^{-6}\right)$. Using effect sizes corrected for winner's curse ${ }^{27,28}$ for each of the 19 variants that were genome-wide significant in the GWAS, we calculated power to achieve significant results (1-tailed $\mathrm{p}<0.05$ ) in the follow-up samples or genome-wide significance in combined analysis (Supplementary Note, Supplementary Table 6, Supplementary Figure 4). We found that the number of variants significant in follow-up is close to expectation ( 8 observed with follow-up $\mathrm{p}<0.05,8.26$ expected, Poisson binomial $\mathrm{p}=$ 0.57 ), and that 11 variants achieving genome-wide significance in the combined analysis is also within the expected range $(p=0.29)$. As an alternative to winner's curse correction, we conducted a polygenic inference analysis using a mixture of Gaussian effect size distributions to model BD genetic architecture and estimate the variants' true effect sizes ${ }^{29}$ (Supplementary Note, Supplementary Figure 5). Under this model, we found that only two variants were nominally significantly weaker in follow-up than expected by chance ( TRANK1 rs9834970 p = 0.012, and rs13821 p = 0.026; Supplementary Table 7), and none were Bonferroni significant ( $p>0.05 / 19=0.0026$ ). Thus, the overall replication rate is within the expected range given the polygenic architecture of $\mathrm{BD}$. 
We next asked if the variants tested in the follow-up samples were, in aggregate, consistent with the presence of additional sub genome-wide significant BD association signals. After excluding 47 variants that were genome-wide significant in our GWAS, our combined analysis or previous BD GWAS, 775 variants remained in our follow-up experiment. 551 variants had the same direction of effect in the discovery GWAS and follow-up (71\%, compared to a null expectation of $50 \%$, sign test $\left.\mathrm{p}=1.3 \times 10^{-32}\right)$, and 110 variants had the same direction of effect and were nominally significant $(\mathrm{p}<0.05)$ in the follow-up $(14 \%$, compared to an expected value of $5 \%$, binomial test $\mathrm{p}=2.1 \times 10^{-22}$ ). This consistency between our GWAS and follow-up results suggests that many more true BD associations exist among these variants.

To identify additional independent signals, we conducted conditional analyses across each of the 30 significant BD loci (Supplementary Table 8). We used the effective number of independent variants based on LD structure within loci ${ }^{30}$ to calculate a multiple testcorrected significance threshold ( $\mathrm{p}=1.01 \times 10^{-5}$, see Supplementary Note). Only one locus showed evidence for an independent association signal (rs114534140 in locus \#8, FSTL5; $\left.\mathrm{p}_{\text {conditional }}=2 \times 10^{-6}\right)$. At another locus (\#30,STK4 on chr 20), we found two SNPs with genome-wide significance in low $\mathrm{LD}\left(\mathrm{R}^{2}<0.1\right)$; however, conditional analysis showed that their associations were not independent.

\section{Shared loci and genetic correlations with other traits}

We next examined the genetic relationships of $\mathrm{BD}$ to other psychiatric disorders and traits. Of the 30 genome-wide significant BD loci, 8 also harbor schizophrenia (SCZ) associations 31,32 . Based on conditional analyses, the BD and SCZ associations appear to be independent at 3 of the 8 shared loci (NCAN, TRANK1 and chr7q22.3:105Mb) (Supplementary Table 9). No genome-wide significant BD locus overlapped with those identified for depression (DEPR), including 44 risk loci identified in the most recent PGC GWAS of major depression ${ }^{33}$, and those reported in a large study of depressive symptoms or subjective well-being ${ }^{34}$. As previously reported ${ }^{35}$, we found substantial and highly significant genetic correlations between $\mathrm{BD}$ and $\mathrm{SCZ}{ }^{31}$ (LD-score regression estimated genetic correlation $\mathrm{r}_{\mathrm{g}}=0.70$, se = $0.020)$ and between BD and DEPR ${ }^{33}\left(r_{\mathrm{g}}=0.35\right.$, se $\left.=0.026\right)$. The BD and DEPR genetic correlation was similar to that observed for $\mathrm{SCZ}$ and DEPR $\left(\mathrm{r}_{\mathrm{g}}=0.34\right.$, se $\left.=0.025\right)$ (Supplementary Table 10A).

We found significant genetic correlations between $\mathrm{BD}$ and other psychiatric-relevant traits (Supplementary Table 10B), including autism spectrum disorder ${ }^{8}\left(\mathrm{r}_{\mathrm{g}}=0.18, \mathrm{P}=2 \times 10^{-4}\right)$, anorexia nervosa $^{36}\left(\mathrm{r}_{\mathrm{g}}=0.23, \mathrm{P}=9 \times 10^{-8}\right)$, and subjective well-being ${ }^{34}\left(\mathrm{r}_{\mathrm{g}}=-0.22\right.$, $\left.\mathrm{P}=4 \times 10^{-7}\right)$. There was suggestive positive overlap with anxiety disorders $\left(\mathrm{r}_{\mathrm{g}}=0.21, \mathrm{P}=0.04\right)$

37 and neuroticism $\left(r_{g}=0.12, P=0.002\right)^{38}$. Significant $r_{g}$ s were seen with measures of education: college attendance ${ }^{39}\left(r_{\mathrm{g}}=0.21, \mathrm{P}=1=\mathrm{x} 10^{-7}\right)$ and education years ${ }^{40}\left(\mathrm{r}_{\mathrm{g}}=0.20\right.$, $\left.\mathrm{P}=6 \times 10^{-14}\right)$, but not with childhood $\mathrm{IQ}^{41}\left(\mathrm{r}_{\mathrm{g}}=0.05, \mathrm{P}=0.5\right)$ or intelligence ${ }^{42}\left(\mathrm{r}_{\mathrm{g}}=-0.05\right.$, $\mathrm{P}=0.08$ ). Among a large number of variants in BD risk loci that were associated with additional traits in the GWAS catalog ${ }^{43}$, we found a handful of loci with non-independent associations (in one overlapping locus with each of educational attainment, biliary atresia, bone mineral density, lipid-related biomarkers) (Supplementary Table 9). Biliary atresia and 
lipid- related biomarkers, however, did not show significant genetic correlation with BD

(Supplementary Table 10B).

\section{BD subtypes}

We performed a secondary GWAS focusing on three clinically recognized subtypes of bipolar disorder: BD1 ( $\mathrm{n}=14,879$ cases), BD2 ( $\mathrm{n}=3,421$ cases), and SAB ( $\mathrm{n}=977$ cases) (Supplementary Note, Supplementary Tables 1A \& 11, Supplementary Figure 6). We observed variants in 14 loci with genome-wide significance for BD1, 10 of which were in genome-wide significant loci in the combined BD GWAS analysis. Not surprisingly given the sample overlap, 3 of the 4 remaining loci genome-wide significant for BD1 have $\mathrm{P}<$ $10^{-6}$ in either our discovery GWAS or combined analysis. The remaining locus (MAD1L1, chr7:1.9Mb, discovery GWAS $\mathrm{p}=2.4 \times 10^{-6}$ ) was recently published in two BD GWAS that included Asian samples 44,45 . We did not observe genome-wide significant results for the smaller BD2 and SAB analyses. BD1, BD2 and SAB all have significant common variant heritabilities $\left(B D 1 \mathrm{~h}^{2}{ }_{\mathrm{snp}}=0.25\right.$, se $=0.014, \mathrm{p}=3.2 \times 10^{-77} ; \mathrm{BD} 2 \mathrm{~h}^{2}{ }_{\mathrm{snp}}=0.11, \mathrm{se}=0.028, \mathrm{p}$ $=5.8 \times 10^{-5} ; \mathrm{SAB} \mathrm{h}_{\text {snp }}^{2}=0.25$, se $\left.=0.10, \mathrm{p}=0.0071\right)$. Genetic correlations among $\mathrm{BD}$ subtypes show that these represent closely related, yet partially distinct, phenotypes (Supplementary Table 12).

We conducted polygenic risk score (PRS) analyses to explore the relationship between genetic risk of SCZ and DEPR, and BD subtypes and psychosis (Figure 2, Supplementary Table 13). PRS calculated from SCZ ${ }^{31}$ were significantly higher in BD1 cases than in BD2 cases $\left(\mathrm{p}=5.6 \times 10^{-17}, \mathrm{P}\right.$ threshold $\left.=0.1\right)$ and in cases with psychosis compared to those without psychosis $\left(\mathrm{p}=2.12 \times 10^{-6}, \mathrm{P}\right.$ threshold $\left.=0.1\right)$. Conversely, $\mathrm{PRS}$ calculated from DEPR ${ }^{33}$ were significantly higher in $\mathrm{BD} 2$ cases than in $\mathrm{BD} 1$ cases $\left(\mathrm{P}=8.5 \times 10^{-10}\right.$, $\mathrm{P}$ threshold $=$ 0.01 ), independent of psychosis. Genetic correlations from LD-score regression support these results; genetic correlations were greater for SCZ with BD1 $\left(\mathrm{r}_{\mathrm{g}}=0.71\right.$, se $\left.=0.025\right)$ than with BD2 $\left(r_{\mathrm{g}}=0.51, \mathrm{se}=0.072\right)$, and were greater for DEPR with BD2 $\left(\mathrm{r}_{\mathrm{g}}=0.69\right.$, se = $0.093)$ than with BD1 $\left(r_{\mathrm{g}}=0.30, \mathrm{se}=0.028\right)($ Supplementary Table 12$)$.

\section{Systems biology and in silico functional analyses}

We tested for functional genomic enrichment in our BD GWAS using partitioned LD-score regression and a range of functional annotations across tissues ${ }^{46}$ (Supplementary Note, Supplementary Table 14). SNP-based BD heritability was most enriched in open chromatin annotations in the central nervous system (proportion SNPs $=0.14$, proportion $\mathrm{h}^{2}{ }_{\mathrm{snp}}=0.60$, enrichment $=3.8, \mathrm{p}=3 \times 10^{-14}$ ). We also used DEPICT ${ }^{47}$ to test for expression of BDassociated genes across tissues, and found significant enrichment of central nervous system $\left(\mathrm{p}<1.4 \times 10^{-3}, \mathrm{FDR}<0.01\right)$ and neurosecretory system $\left(\mathrm{p}=2.0 \times 10^{-6}, \mathrm{FDR}<0.01\right)$ genes (Supplementary Table 15).

To prioritize genes that may play a functional role in BD, we integrated BD GWAS association statistics with eQTL (SNP-gene expression association) and mQTL (SNP-DNA methylation association) data using summary Mendelian randomization (SMR) 48,49,50 (Supplementary Table 16; Supplementary Note). SMR identified 21 genes using eQTL data that were significant after multiple testing correction, without evidence of heterogeneity 
between GWAS and eQTL association signals. Association with GNL3 was observed in both brain and blood, highlighting the utility of using blood eQTL data as proxy for brain eQTLs ${ }^{50}$. Methylation profiles at $6 \mathrm{CpGs}$ in brain and $10 \mathrm{CpGs}$ in blood were associated with BD, four of which overlapped between brain and blood mQTL: MUSTN1, GLT8D1, HAPLN4 and FADS2.

Finally, we used MAGMA ${ }^{51}$ to conduct a gene-wise BD GWAS and to test for enrichment of pathways curated from multiple sources (see Supplementary Note). We note that significance levels were assigned to genes by physical proximity of SNPs, and do not imply that significant genes are causal for BD. Genic association results included 154 Bonferroni significant genes (MAGMA pJOINT $<2.8 \times 10^{-6}$ ), including 82 genes in 20 genome-wide significant loci, and 73 genes in 27 additional loci that did not reach genome-wide significance (Supplementary Table 17). Nine related pathways were significantly enriched for genes with BD associations ( $\mathrm{p}<7.0 \times 10^{-5}$, FDR $<0.05$ ), including abnormal motor coordination/balance pathways (from mice), regulation of insulin secretion, and endocannabinoid signaling (Supplementary Table 18, Supplementary Figure 7).

\section{DISCUSSION}

We carried out a large bipolar disorder (BD) GWAS and identified 30 genome-wide significant loci, including 20 that were novel. Previous BD GWAS have reported a total of 20 loci significantly associated with $\mathrm{BD}^{9-23}$; twelve of these previously reported loci were not genome-wide significant in our GWAS meta analysis, but all had $\mathrm{P}_{\mathrm{GWAS}} \leq 1.3 \times 10^{-5}$ (Supplementary Table 4C). Our recent GWAS of BD and SCZ ${ }^{52}$, which included our discovery GWAS data jointly analyzed with published SCZ data ${ }^{31}$ (without overlapping control subjects), highlighted similarities and differences in BD and SCZ in terms of known associated SNPs and PRS-subphenotype associations; here we maximized power to identify $\mathrm{BD}$ associations. Phenotypic variance explained by polygenic risk scores (PRS) based on our BD GWAS data is $\sim 8 \%$ (observed scale; $4 \%$ on the liability scale ${ }^{53}$ ), an increase from $2.8 \%$ ( $1.2 \%$ on the liability scale) in our previous study ${ }^{9}$. The results of our BD subtype PRS analyses support the nosological distinction between BD1 and BD2, but also highlight the importance of psychosis beyond DSM subtypes, corroborating and expanding evidence from previous clinical ${ }^{54}$ and genetic studies ${ }^{52,55,56}$. The DEPR vs. BD PRS analyses provide further support for the distinction between BD1 and BD2, independent of the presence of psychosis.

Of the 19 loci identified in our discovery GWAS, only 11 were genome-wide significant in meta-analysis of our GWAS and follow-up samples. These results are not unexpected given small effect sizes, the winner's curse ${ }^{28,57}$ (Supplementary Note and Supplementary Figure 4); SNPs can teeter-totter around the genome-wide significance threshold even as sample sizes increase. Genetic heterogeneity observed among BD GWAS cohorts ${ }^{8}$ could also contribute to inconsistent replication results; we observed variable polygenic effects between BD subtypes (Figure 2, Supplementary Table 13) as well as between cohorts in our study (Supplementary Figure 2, Supplementary Table 4) which used a diversity of criteria to define cases (Supplementary Note). Remarkably, the strongest association signal from the discovery GWAS, at the TRANK1 locus (rs9834970; $\mathrm{p}_{\text {combined }}=5.7 \mathrm{E}-12, \mathrm{OR}=0.93$ ), 
exhibited significant heterogeneity among discovery GWAS cohorts (Cochran's Q $\mathrm{p}=$ $\left.1.9 \times 10^{-4}\right)$, and did not replicate in the follow-up sample (1-tailed $\left.\mathrm{p}_{\text {followup }}=0.3\right)$ (Supplementary Data $2 \& 3$ ). This locus has been significant in recent ${ }^{11,12,17,18}$ but not earlier BD GWAS $9,13,20$. Thus, complex genetic architecture as well as phenotypic heterogeneity may contribute to the inconsistency of genome-wide significant findings within and across BD GWAS studies. The observed heterogeneity is a major challenge for GWAS of psychiatric disorders and calls for careful and systematic clinical assessment of cases and controls in parallel with continued efforts to collect larger sample sizes.

Of the $30 \mathrm{BD}$ associated loci, 8 also harbor associations $31,32,58$ with schizophrenia (SCZ); however, conditional analyses suggest that the $\mathrm{BD}$ and $\mathrm{SCZ}$ associations at 3 of the 8 shared loci (in the NCAN, TRANK1 and chr7q22.3:105 Mb loci) may be independent (Supplementary Table 9). Differential BD and SCZ associations may represent opportunities to understand the genetic distinctions between these closely related and sometimes clinically difficult to distinguish disorders. We did not find BD loci that overlap with those associated with major depression ${ }^{33}$.

The confirmed association within loci containing $C A C N A 1 C$ and other voltage-gated calcium channel genes supports the rekindled interest in calcium channel antagonists as potential treatments for $\mathrm{BD}$, with similar examination ongoing for other genes implicated in SCZ GWAS ${ }^{59}$. Other genes within novel BD-associated loci include those coding for other ion channels and transporters (SCN2A, SLC4A1), neurotransmitter receptors (GRIN2A) and synaptic components (RIMS1, ANK3). Further study will confirm whether or not these are the causal genes in the loci. These processes are important in neuronal hyperexcitability ${ }^{60}$, an excess of which has been reported in iPSC derived neurons from BD patients, and which has been shown to be affected by the classic mood stabilizing drug lithium ${ }^{61}$. In addition, SMR eQTL and mQTL analyses implicate GLT8D1, which is involved in proliferation and differentiation of neural stem cells ${ }^{62}$. Pathway analyses reveal new genetic evidence for insulin secretion and endocannabinoid signaling in BD. There is evidence of insulin action in the brain ${ }^{63}$ and in $\mathrm{BD}^{64}$. The endocannabinoid system has possible roles in schizophrenia ${ }^{65,66}$ and depression ${ }^{67}$. Top genes appearing in these pathways include calcium and potassium channel subunit, MAP kinase and GABA-A receptor subunit genes (Supplementary Table 18).

We observe significant positive genetic correlations with educational attainment, but not with either adult or childhood IQ, suggesting that the role of BD genetics in educational attainment may be independent of general intelligence. This result is inconsistent with suggestions from epidemiological studies ${ }^{68}$, but in agreement with a recent clinical study ${ }^{69}$.

In summary, findings from the genome-wide analysis of $\mathrm{BD}$ reveal an extensive polygenic genetic architecture of the disease, implicate brain calcium channels and neurotransmitter function in BD etiology, and confirm that BD is part of a spectrum of highly correlated psychiatric and mood disorders. 


\section{Accession codes:}

Accessioned data were part of the following datasets analyzed in this study. Fat2: phs000167.v1.p1 or PGC bundle phs001254.v1.p1 (MGS nonGAIN controls). Gain: dbGAP phs000017.v3.p1 (GAIN Bip cases/controls), phs000021.v3.p2 (GAIN scz controls); also part of the PGC dbGAP bundle phs001254.v1.p1. Jjst: phs000092.v1.p1 or PGC bundle phs001254.v1.p1 (SAGE controls). St2c, NIMH RGR Bipolar Study 19 (STEP-BD), dbGAP phs000294.v1.p1 (MIGEN controls). Mich: NIMH RGR Bipolar Study 2 (Pritzker). Wtcc: EGAD00000000002.

\section{Data Availability.}

The PGC's policy is to make genome-wide summary results public. Summary statistics for our meta-analysis are available through the PGC (https://www.med.unc.edu/pgc/results-anddownloads). Data are accessible with collaborative analysis proposals through the Bipolar Disorder working group of the PGC (https://med.unc.edu/pgc).

\section{ONLINE METHODS}

\section{GWAS and follow-up cohorts.}

Our discovery GWAS sample was comprised of 32 cohorts from 14 countries in Europe, North America and Australia (Supplementary Table 1A), totaling 20,352 cases and 31,358 controls of European descent. A selected set of variants (see below) were tested in 7 followup cohorts of European descent (Supplementary Table 1B), totalling 9,025 cases and 142,824 controls $\left(\mathrm{N}_{\mathrm{eff}}=23,991\right)$. The Supplementary Note summarizes the source and inclusion/exclusion criteria for cases and controls for each cohort. All cohorts in the initial PGC BD paper were included ${ }^{9}$. Cases were required to meet international consensus criteria (DSM-IV or ICD-10) for a lifetime diagnosis of BD established using structured diagnostic instruments from assessments by trained interviewers, clinician-administered checklists, or medical record review. In most cohorts, controls were screened for the absence of lifetime psychiatric disorders and randomly selected from the population.

\section{GWAS cohort analysis}

We tested 20 principal components for association with BD using logistic regression; seven were significantly associated with phenotype and used in GWAS association analysis (PCs 1-6, 19). In each cohort, we performed logistic regression association tests for BD with imputed marker dosages including 7 principal components to control for population stratification. For all GWAS cohorts, X-chromosome association analyses were conducted separately by sex, and then meta-analyzed across sexes. We also conducted BD1, BD2, and SAB GWAS, retaining only cohorts with at least 30 subtype cases and filtering SNPs for MAF $>0.02$. Results were combined across cohorts using an inverse variance-weighted fixed effects meta-analysis ${ }^{70}$. We used Plink 'clumping' ${ }^{71,72}$ to identify an LD-pruned set of discovery GWAS meta-analysis BD-associated variants $(P<0.0001$, and distance $>500$ $\mathrm{kb}$ or $\mathrm{LD} \mathrm{r} \mathrm{r}^{2}<0.1, \mathrm{n}$ variants $=822$ ) for analysis in the follow-up cohorts. Conditional analyses were conducted within each GWAS cohort and meta-analyzed as above. 


\section{Follow-up cohort analysis.}

In each follow-up cohort we performed BD association analysis of the 822 selected GWAS variants (when available) including genetic ancestry covariates, following $\mathrm{QC}$ and analysis methods of the individual study contributors. We performed inverse variance-weighted fixed-effects meta-analyses of the association results from the follow-up cohorts, and of the discovery GWAS and follow-up analyses.

\section{Polygenic risk score (PRS) analyses.}

We tested PRS for our primary GWAS on each GWAS cohort as a target set, using a GWAS where the target cohort was left out of the meta-analysis (Supplementary Table 2). To test genetic overlaps with other psychiatric diseases, we calculated PRS for DEPR and SCZ in our GWAS cohort BD cases ${ }^{73}$. In pairwise case subtype or psychosis analyses (Figure 2, Supplementary Table 13), we regressed outcome on the PRS adjusting for ancestry principal components and a cohort indicator using logistic regression, and visualized covariateadjusted PRS in BD1 and BD2 subtypes (Figure 2). Outcome sample sizes were BD1 $n=8,044, B D 2 n=3,365, S A B n=977$; BD1 cases with and without psychosis $n=2175$ and 798 respectively, BD2 cases with and without psychosis $n=146$ and 660 .

\section{Linkage disequilibrium (LD) score regression.}

LD score regression ${ }^{25,26}$ was used to conduct SNP-heritability analyses from GWAS summary statistics. LD score regression bivariate genetic correlations attributable to genome-wide common variants were estimated between the full BD GWAS, BD subtype GWASs, and other traits and disorders in LD-Hub ${ }^{26}$. We also used LD score regression to partition heritability by genomic features 46 .

\section{Relation of BD GWA findings to tissue and cellular gene expression.}

We used partitioned LD score ${ }^{47,74}$ and DEPICT ${ }^{47}$ regression to evaluate which somatic tissues and brain tissues were enriched in the BD GWAS. We used summary-data-based Mendelian randomization (SMR) ${ }^{48,50}$ to identify SNPs with strong evidence of causality of brain or blood gene expression or methylation in BD risk (Supplementary Table 16), with a test for heterogeneity to exclude regions with LD between distinct causal SNPs (pHET < $0.01)$.

\section{Gene-wise and pathway analysis.}

Guided by rigorous method comparisons conducted by PGC members ${ }^{51,75}$, p-values quantifying the degree of association of genes and gene sets with BD were generated using MAGMA (v1.06) ${ }^{51}$. We used ENSEMBL gene coordinates for 18,172 genes giving a Bonferroni corrected $P$-value threshold of $2.8 \times 10^{-6}$. Joint multi-SNP LD-adjusted genelevel p-values were calculated using SNPs $35 \mathrm{~kb}$ upstream to $10 \mathrm{~kb}$ downstream, adjusting for LD using 1,000 Genomes Project (Phase 3 v5a, MAF $\geq 0.01$, European-ancestry subjects) ${ }^{76}$. Gene sets were compiled from multiple sources. Competitive gene set tests were conducted correcting for gene size, variant density, and LD within and between genes. The pathway map (Supplementary Figure 5) was constructed using the kernel generative topographic mapping algorithm (k-GTM) as described by ${ }^{77}$. 
Genome build.

All genomic coordinates are given in NCBI Build 37/UCSC hg19.

\section{Supplementary Material}

Refer to Web version on PubMed Central for supplementary material.

\section{Authors}

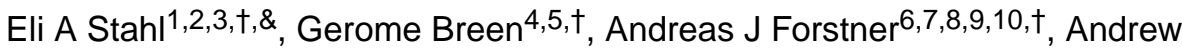
McQuillin ${ }^{11, \dagger}$, Stephan Ripke ${ }^{12,13,14, \dagger}$, Vassily Trubetskoy ${ }^{13}$, Manuel Mattheisen 15,16,17,18,19, Yunpeng Wang ${ }^{20,21}$, Jonathan R I Coleman ${ }^{4,5}$, Héléna A Gaspar $^{4,5}$, Christiaan A de Leeuw ${ }^{22}$, Stacy Steinberg ${ }^{23}$, Jennifer M Whitehead Pavlides $^{24}$, Maciej Trzaskowski ${ }^{25}$, Enda M Byrne ${ }^{25}$, Tune H Pers ${ }^{3,26}$, Peter A Holmans $^{27}$, Alexander L Richards ${ }^{27}$, Liam Abbott ${ }^{12}$, Esben Agerbo ${ }^{19,28,29}$, Huda Akil $^{30}$, Diego Albani ${ }^{31}$, Ney Alliey-Rodriguez ${ }^{32}$, Thomas D Als ${ }^{15,16,19}$, Adebayo Anjorin ${ }^{33}$, Verneri Antilla ${ }^{14}$, Swapnil Awasthi ${ }^{13}$, Judith A Badner ${ }^{34}$, Marie BækvadHansen ${ }^{19,35}$, Jack D Barchas ${ }^{36}$, Nicholas Bass ${ }^{11}$, Michael Bauer ${ }^{37}$, Richard Belliveau $^{12}$, Sarah E Bergen ${ }^{38}$, Carsten Bøcker Pedersen ${ }^{19,28,29}$, Erlend Bøen ${ }^{39}$, Marco P. Boks ${ }^{40}$, James Boocock ${ }^{41}$, Monika Budde ${ }^{42}$, William Bunney ${ }^{43}$, Margit Burmeister $^{44}$, Jonas Bybjerg-Grauholm ${ }^{19,35}$, William Byerley ${ }^{45}$, Miquel Casas $^{46,47,48,49}$, Felecia Cerrato ${ }^{12}$, Pablo Cervantes ${ }^{50}$, Kimberly Chambert ${ }^{12}$, Alexander W Charney ${ }^{2}$, Danfeng Chen ${ }^{12}$, Claire Churchhouse ${ }^{12,14}$, Toni-Kim Clarke $^{51}$, William Coryell ${ }^{52}$, David W Craig ${ }^{53}$, Cristiana Cruceanu ${ }^{50,54}$, David Curtis $^{55,56}$, Piotr M Czerski ${ }^{57}$, Anders M Dale $58,59,60,61$, Simone de Jong ${ }^{4,5}$, Franziska Degenhardt ${ }^{8}$, Jurgen Del-Favero ${ }^{62}$, J Raymond DePaulo ${ }^{63}$, Srdjan Djurovic $^{64,65}$, Amanda L Dobbyn ${ }^{1,2}$, Ashley Dumont ${ }^{12}$, Torbjørn Elvsåshagen ${ }^{66,67}$, Valentina Escott-Price ${ }^{27}$, Chun Chieh Fan ${ }^{61}$, Sascha B Fischer6,10, Matthew Flickinger $^{68}$, Tatiana M Foroud ${ }^{69}$, Liz Forty ${ }^{27}$, Josef Frank ${ }^{70}$, Christine Fraser ${ }^{27}$, Nelson B Freimer ${ }^{71}$, Louise Frisén ${ }^{72,73,74}$, Katrin Gade ${ }^{42,75}$, Diane Gage ${ }^{12}$, Julie Garnham $^{76}$, Claudia Giambartolomei ${ }^{77}$, Marianne Giørtz Pedersen ${ }^{19,28,29}$, Jaqueline Goldstein ${ }^{12}$, Scott D Gordon ${ }^{78}$, Katherine Gordon-Smith ${ }^{79}$, Elaine K Green $^{80}$, Melissa J Green ${ }^{81,82}$, Tiffany A Greenwood ${ }^{60}$, Jakob Grove ${ }^{15,16,19,83,}$ Weihua Guan ${ }^{84}$, José Guzman-Parra ${ }^{85}$, Marian L Hamshere ${ }^{27}$, Martin Hautzinger ${ }^{86}$, Urs Heilbronner ${ }^{42}$, Stefan Herms ${ }^{6,8,10}$, Maria Hipolito ${ }^{87}$, Per Hoffmann $^{6,8,10}$, Dominic Holland ${ }^{58,88}$, Laura Huckins ${ }^{1,2}$, Stéphane Jamain ${ }^{89,90,}$ Jessica S Johnson ${ }^{1,2}$, Anders Juréus ${ }^{38}$, Radhika Kandaswamy ${ }^{4}$, Robert Karlsson ${ }^{38}$, James L Kennedy ${ }^{91,92,93,94}$, Sarah Kittel-Schneider ${ }^{95}$, James A Knowles ${ }^{96,97}$, Manolis Kogevinas ${ }^{98}$, Anna C Koller ${ }^{8}$, Ralph Kupka99,100,101, Catharina Lavebratt ${ }^{72}$, Jacob Lawrence ${ }^{102}$, William B Lawson ${ }^{87}$, Markus Leber ${ }^{103}$, Phil H Lee 12,14,104, Shawn E Levy ${ }^{105}$, Jun Z Li ${ }^{106}$, Chunyu Liu ${ }^{107}$, Susanne Lucae ${ }^{108}$, Anna Maaser ${ }^{8}$, Donald J Maclntyre ${ }^{109,110}$, Pamela B Mahon 63,111 , Wolfgang Maier ${ }^{112}$, Lina Martinsson ${ }^{73}$, Steve McCarroll ${ }^{12,113}$, Peter McGuffin ${ }^{4}$, Melvin G Mclnnis ${ }^{114}$, James D McKay ${ }^{115}$, Helena Medeiros ${ }^{97}$, Sarah E Medland ${ }^{78}$, Fan Meng ${ }^{30,114}$, Lili Milani ${ }^{116}$, Grant W Montgomery ${ }^{25}$, Derek W Morris ${ }^{117,118}$, Thomas W 
Mühleisen 6,119 , Niamh Mullins ${ }^{4}$, Hoang Nguyen ${ }^{1,2}$, Caroline M Nievergelt60,120, Annelie Nordin Adolfsson ${ }^{121}$, Evaristus A Nwulia ${ }^{87}$, Claire ODonovan ${ }^{76}$, Loes M Olde Loohuis ${ }^{71}$, Anil P S Ori ${ }^{71}$, Lilijana Oruc ${ }^{122}$, Urban Ösby ${ }^{123}$, Roy H Perlis ${ }^{124,125}$, Amy Perry ${ }^{79}$, Andrea Pfennig ${ }^{37}$, James B Potash 63 , Shaun M Purcell2,111, Eline J Regeer ${ }^{126}$, Andreas Reif ${ }^{95}$, Céline S Reinbold ${ }^{6,10}$, John P Rice $^{127}$, Fabio Rivas ${ }^{85}$, Margarita Rivera ${ }^{4,128}$, Panos Roussos ${ }^{1,2,129}$, Douglas M Ruderfer ${ }^{130}$, Euijung Ryu ${ }^{131}$, Cristina Sánchez-Mora ${ }^{46,47,49}$, Alan F Schatzberg ${ }^{132}$, William A Scheftner ${ }^{133}$, Nicholas J Schork ${ }^{134}$, Cynthia Shannon Weickert ${ }^{81,82}$, Tatyana Shehktman ${ }^{60}$, Paul D Shilling ${ }^{60}$, Engilbert Sigurdsson ${ }^{135}$, Claire Slaney ${ }^{76}$, Olav B Smeland ${ }^{136,137}$, Janet L Sobell ${ }^{138}$, Christine Søholm Hansen ${ }^{19,35}$, Anne T Spijker $^{139}$, David St Clair ${ }^{140}$, Michael Steffens ${ }^{141}$, John S Strauss ${ }^{93,142}$, Fabian Streit $^{70}$, Jana Strohmaier ${ }^{70}$, Szabolcs Szelinger ${ }^{143}$, Robert C Thompson ${ }^{114}$, Thorgeir E Thorgeirsson ${ }^{23}$, Jens Treutlein ${ }^{70}$, Helmut Vedder ${ }^{144}$, Weiqing Wang ${ }^{1,2}$, Stanley J Watson ${ }^{114}$, Thomas W Weickert81,82, Stephanie H Witt ${ }^{70}$, Simon Xi ${ }^{145}$, Wei Xu ${ }^{146,147}$, Allan $\mathrm{H}$ Young ${ }^{148}$, Peter Zandi ${ }^{149}$, Peng Zhang ${ }^{150}$, Sebastian Zöllner $^{114}$, eQTLGen Consortium ${ }^{151}$, BIOS Consortium ${ }^{151}$, Rolf Adolfsson ${ }^{121}$, Ingrid Agartz 17,39,152, Martin Alda76,153, Lena Backlund ${ }^{73}$, Bernhard T Baune ${ }^{154,155}$, Frank Bellivier ${ }^{156,157,158,159}$, Wade H Berrettini ${ }^{160}$, Joanna M Biernacka $^{131}$, Douglas H R Blackwood ${ }^{51}$, Michael Boehnke ${ }^{68}$, Anders D Børglum ${ }^{15,16,19}$, Aiden Corvin ${ }^{118}$, Nicholas Craddock ${ }^{27}$, Mark J Daly ${ }^{12,14}$, Udo Dannlowski ${ }^{155}$, Tõnu Esko3,113,116,161, Bruno Etain ${ }^{156,158,159,162}$, Mark Frye ${ }^{163}$, Janice M Fullerton ${ }^{82,164}$, Elliot S Gershon ${ }^{32,165}$, Michael Gill ${ }^{118}$, Fernando Goes ${ }^{63}$, Maria Grigoroiu-Serbanescu ${ }^{166}$, Joanna Hauser ${ }^{57}$, David M Hougaard ${ }^{19,35}$, Christina M Hultman ${ }^{38}$, lan Jones ${ }^{27}$, Lisa A Jones ${ }^{79}$, René S Kahnn ${ }^{2,40}$, George Kirov 27 , Mikael Landén 38,167 , Marion Leboyer ${ }^{90,156,168}$, Cathryn M Lewis ${ }^{4,5,169}$, Qingqin S Li ${ }^{170}$, Jolanta Lissowska ${ }^{171}$, Nicholas G Martin ${ }^{78,172}$, Fermin Mayoral ${ }^{85}$, Susan L McElroy ${ }^{173}$, Andrew M McIntosh ${ }^{51,174}$, Francis J McMahon ${ }^{175}$, Ingrid Melle ${ }^{176,177}$, Andres Metspalu116,178, Philip B Mitchell ${ }^{81}$, Gunnar Morken 179,180, Ole Mors ${ }^{19,181}$, Preben Bo Mortensen ${ }^{15,19,28,29}$, Bertram Müller-Myhsok ${ }^{54,182,183}$, Richard M Myers ${ }^{105}$, Benjamin M Neale $3,12,14$, Vishwajit Nimgaonkar ${ }^{184}$, Merete Nordentoft ${ }^{19,185}$, Markus M Nöthen ${ }^{8}$, Michael C ODonovan ${ }^{27}$, Ketil J Oedegaard 186,187, Michael J Owen²7, Sara A Paciga ${ }^{188}$, Carlos Pato ${ }^{97,189}$, Michele T Pato ${ }^{97}$, Danielle Posthuma 22,190 , Josep Antoni Ramos-Quiroga $46,47,48,49$, Marta Ribasés ${ }^{46,47,49}$, Marcella Rietschel ${ }^{70}$, Guy A Rouleau ${ }^{191,192}$, Martin Schalling ${ }^{72}$, Peter R Schofield 82,164 , Thomas G Schulze ${ }^{42,63,70,75,175}$, Alessandro Serretti ${ }^{193}$, Jordan W Smoller ${ }^{12,194,195}$, Hreinn Stefansson ${ }^{23}$, Kari Stefansson ${ }^{23,196}$, Eystein Stordal ${ }^{197,198}$, Patrick F Sullivan ${ }^{38,199,200}$, Gustavo Turecki201, Arne E Vaaler ${ }^{202}$, Eduard Vieta ${ }^{203}$, John B Vincent ${ }^{142}$, Thomas Werge ${ }^{19,204,205}$, John I

Nurnberger ${ }^{206}$, Naomi R Wray ${ }^{24,25}$, Arianna Di Florio ${ }^{27,200, ~ H o w a r d ~ J ~ E d e n b e r g ~}{ }^{207}$, Sven Cichon 6,8,10,119, Roel A Ophoff $40,41,71$, Laura J Scott 68 , Ole A Andreassen ${ }^{136,137}$, John Kelsoe ${ }^{60,{ }^{*}, \&}$, Pamela Sklar ${ }^{1,2,{ }^{*}}$, Bipolar Disorder Working Group of the Psychiatric Genomics Consortium ${ }^{151}$ 


\section{Affiliations}

${ }^{1}$ Department of Genetics and Genomic Sciences, Icahn School of Medicine at Mount Sinai, New York, NY, US

${ }^{2}$ Department of Psychiatry, Icahn School of Medicine at Mount Sinai, New York, NY, US

${ }^{3}$ Medical and Population Genetics, Broad Institute, Cambridge, MA, US

${ }^{4} \mathrm{MRC}$ Social, Genetic and Developmental Psychiatry Centre, King's College London, London, GB

${ }^{5}$ NIHR BRC for Mental Health, King's College London, London, GB

${ }^{6}$ Department of Biomedicine, University of Basel, Basel, $\mathrm{CH}$

${ }^{7}$ Department of Psychiatry (UPK), University of Basel, Basel, $\mathrm{CH}$

${ }^{8}$ Institute of Human Genetics, University of Bonn School of Medicine \& University Hospital Bonn, Bonn, DE

${ }^{9}$ Centre for Human Genetics, University of Marburg, Marburg, DE

${ }^{10}$ Institute of Medical Genetics and Pathology, University Hospital Basel, Basel, $\mathrm{CH}$

${ }^{11}$ Division of Psychiatry, University College London, London, GB

${ }^{12}$ Stanley Center for Psychiatric Research, Broad Institute, Cambridge, MA, US

${ }^{13}$ Department of Psychiatry and Psychotherapy, Charité - Universitätsmedizin, Berlin, DE

${ }^{14}$ Analytic and Translational Genetics Unit, Massachusetts General Hospital, Boston, MA, US

${ }^{15}$ iSEQ, Center for Integrative Sequencing, Aarhus University, Aarhus, DK

${ }^{16}$ Department of Biomedicine - Human Genetics, Aarhus University, Aarhus, DK

${ }^{17}$ Department of Clinical Neuroscience, Centre for Psychiatry Research, Karolinska Institutet, Stockholm, SE

${ }^{18}$ Department of Psychiatry, Psychosomatics and Psychotherapy, Center of Mental Health, University Hospital Würzburg, Würzburg, DE

${ }^{19}{ }_{\mathrm{iPSYCH}}$, The Lundbeck Foundation Initiative for Integrative Psychiatric Research, DK

${ }^{20}$ Institute of Biological Psychiatry, Mental Health Centre Sct. Hans, Copenhagen, DK

${ }^{21}$ Institute of Clinical Medicine, University of Oslo, Oslo, NO

${ }^{22}$ Department of Complex Trait Genetics, Center for Neurogenomics and Cognitive Research, Amsterdam Neuroscience, Vrije Universiteit Amsterdam, Amsterdam, NL ${ }^{23} \mathrm{deCODE}$ Genetics / Amgen, Reykjavik, IS 
${ }^{24}$ Queensland Brain Institute, The University of Queensland, Brisbane, QLD, AU

${ }^{25}$ Institute for Molecular Bioscience, The University of Queensland, Brisbane, QLD, $\mathrm{AU}$

${ }^{26}$ Division of Endocrinology and Center for Basic and Translational Obesity Research, Boston Children's Hospital, Boston, MA, US

${ }^{27}$ Medical Research Council Centre for Neuropsychiatric Genetics and Genomics, Division of Psychological Medicine and Clinical Neurosciences, Cardiff University, Cardiff, GB

${ }^{28}$ National Centre for Register-Based Research, Aarhus University, Aarhus, DK

${ }^{29}$ Centre for Integrated Register-based Research, Aarhus University, Aarhus, DK

${ }^{30}$ Molecular \& Behavioral Neuroscience Institute, University of Michigan, Ann Arbor, MI, US

${ }^{31}$ NEUROSCIENCE, IRCCS - Istituto Di Ricerche Farmacologiche Mario Negri, Milano, IT

${ }^{32}$ Department of Psychiatry and Behavioral Neuroscience, University of Chicago, Chicago, IL, US

${ }^{33}$ Psychiatry, Berkshire Healthcare NHS Foundation Trust, Bracknell, GB

${ }^{34}$ Psychiatry, Rush University Medical Center, Chicago, IL, US

${ }^{35}$ Center for Neonatal Screening, Department for Congenital Disorders, Statens Serum Institut, Copenhagen, DK

${ }^{36}$ Department of Psychiatry, Weill Cornell Medical College, New York, NY, US

${ }^{37}$ Department of Psychiatry and Psychotherapy, University Hospital Carl Gustav Carus, Technische Universität Dresden, Dresden, DE

${ }^{38}$ Department of Medical Epidemiology and Biostatistics, Karolinska Institutet, Stockholm, SE

${ }^{39}$ Department of Psychiatric Research, Diakonhjemmet Hospital, Oslo, NO

${ }^{40}$ Psychiatry, UMC Utrecht Brain Center Rudolf Magnus, Utrecht, NL

${ }^{41}$ Human Genetics, University of California Los Angeles, Los Angeles, CA, US

${ }^{42}$ Institute of Psychiatric Phenomics and Genomics (IPPG), University Hospital, LMU Munich, Munich, DE

${ }^{43}$ Department of Psychiatry and Human Behavior, University of California, Irvine, Irvine, CA, US

${ }^{44}$ Molecular \& Behavioral Neuroscience Institute and Department of Computational Medicine \& Bioinformatics, University of Michigan, Ann Arbor, MI, US

${ }^{45}$ Psychiatry, University of California San Francisco, San Francisco, CA, US 
${ }^{46}$ Instituto de Salud Carlos III, Biomedical Network Research Centre on Mental Health (CIBERSAM), Madrid, ES

${ }^{47}$ Department of Psychiatry, Hospital Universitari Vall d'Hebron, Barcelona, ES

${ }^{48}$ Department of Psychiatry and Forensic Medicine, Universitat Autònoma de Barcelona, Barcelona, ES

${ }^{49}$ Psychiatric Genetics Unit, Group of Psychiatry Mental Health and Addictions, Vall d'Hebron Research Institut (VHIR), Universitat Autònoma de Barcelona, Barcelona, ES

${ }^{50}$ Department of Psychiatry, Mood Disorders Program, McGill University Health Center, Montreal, QC, CA

${ }^{51}$ Division of Psychiatry, University of Edinburgh, Edinburgh, GB

52University of lowa Hospitals and Clinics, lowa City, IA, US

${ }^{53}$ Translational Genomics, USC, Phoenix, AZ, US

${ }^{54}$ Department of Translational Research in Psychiatry, Max Planck Institute of Psychiatry, Munich, DE

${ }^{55}$ Centre for Psychiatry, Queen Mary University of London, London, GB

${ }^{56}$ UCL Genetics Institute, University College London, London, GB

${ }^{57}$ Department of Psychiatry, Laboratory of Psychiatric Genetics, Poznan University of Medical Sciences, Poznan, PL

${ }^{58}$ Department of Neurosciences, University of California San Diego, La Jolla, CA, US

${ }^{59}$ Department of Radiology, University of California San Diego, La Jolla, CA, US

${ }^{60}$ Department of Psychiatry, University of California San Diego, La Jolla, CA, US

${ }^{61}$ Department of Cognitive Science, University of California San Diego, La Jolla, CA, US

${ }^{62}$ Applied Molecular Genomics Unit, VIB Department of Molecular Genetics, University of Antwerp, Antwerp, Belgium

${ }^{63}$ Department of Psychiatry and Behavioral Sciences, Johns Hopkins University School of Medicine, Baltimore, MD, US

${ }^{64}$ Department of Medical Genetics, Oslo University Hospital Ullevål, Oslo, NO

${ }^{65}$ NORMENT, KG Jebsen Centre for Psychosis Research, Department of Clinical Science, University of Bergen, Bergen, NO

${ }^{66}$ Department of Neurology, Oslo University Hospital, Oslo, NO

${ }^{67}$ NORMENT, KG Jebsen Centre for Psychosis Research, Oslo University Hospital, Oslo, NO 
${ }^{68}$ Center for Statistical Genetics and Department of Biostatistics, University of Michigan, Ann Arbor, MI, US

${ }^{69}$ Department of Medical \& Molecular Genetics, Indiana University, Indianapolis, IN, US

${ }^{70}$ Department of Genetic Epidemiology in Psychiatry, Central Institute of Mental Health, Medical Faculty Mannheim, Heidelberg University, Mannheim, DE

${ }^{71}$ Center for Neurobehavioral Genetics, University of California Los Angeles, Los Angeles, CA, US

${ }^{72}$ Department of Molecular Medicine and Surgery, Karolinska Institutet and Center for Molecular Medicine, Karolinska University Hospital, Stockholm, SE

${ }^{73}$ Department of Clinical Neuroscience, Karolinska Institutet and Center for Molecular Medicine, Karolinska University Hospital, Stockholm, SE

${ }^{74}$ Child and Adolescent Psychiatry Research Center, Stockholm, SE

${ }^{75}$ Department of Psychiatry and Psychotherapy, University Medical Center Göttingen, Göttingen, DE

${ }^{76}$ Department of Psychiatry, Dalhousie University, Halifax, NS, CA

${ }^{77}$ Department of Pathology and Laboratory Medicine, University of California Los Angeles, Los Angeles, CA, US

${ }^{78}$ Genetics and Computational Biology, QIMR Berghofer Medical Research Institute, Brisbane, QLD, AU

${ }^{79}$ Department of Psychological Medicine, University of Worcester, Worcester, GB

${ }^{80}$ School of Biomedical Sciences, Plymouth University Peninsula Schools of Medicine and Dentistry, University of Plymouth, Plymouth, GB

${ }^{81}$ School of Psychiatry, University of New South Wales, Sydney, NSW, AU

${ }^{82}$ Neuroscience Research Australia, Sydney, NSW, AU

${ }^{83}$ Bioinformatics Research Centre, Aarhus University, Aarhus, DK

${ }^{84}$ Biostatistics, University of Minnesota System, Minneapolis, MN, US

${ }^{85}$ Mental Health Department, University Regional Hospital, Biomedicine Institute (IBIMA), Málaga, ES

${ }^{86}$ Department of Psychology, Eberhard Karls Universität Tübingen, Tubingen, DE

${ }^{87}$ Department of Psychiatry and Behavioral Sciences, Howard University Hospital, Washington, DC, US

${ }^{88}$ Center for Multimodal Imaging and Genetics, University of California San Diego, La Jolla, CA, US

${ }^{89}$ Psychiatrie Translationnelle, Inserm U955, Créteil, FR

${ }^{90}$ Faculté de Médecine, Université Paris Est, Créteil, FR 
${ }^{91}$ Campbell Family Mental Health Research Institute, Centre for Addiction and Mental Health, Toronto, ON, CA

${ }^{92}$ Neurogenetics Section, Centre for Addiction and Mental Health, Toronto, ON, CA

${ }^{93}$ Department of Psychiatry, University of Toronto, Toronto, ON, CA

${ }^{94}$ Institute of Medical Sciences, University of Toronto, Toronto, ON, CA

${ }^{95}$ Department of Psychiatry, Psychosomatic Medicine and Psychotherapy, University Hospital Frankfurt, Frankfurt am Main, DE

${ }^{96}$ Cell Biology, SUNY Downstate Medical Center College of Medicine, Brooklyn, NY, US

${ }^{97}$ Institute for Genomic Health, SUNY Downstate Medical Center College of Medicine, Brooklyn, NY, US

${ }^{98}$ ISGlobal, Barcelona, ES

${ }^{99}$ Psychiatry, Altrecht, Utrecht, NL

${ }^{100}$ Psychiatry, GGZ inGeest, Amsterdam, NL

${ }^{101}$ Psychiatry, VU medisch centrum, Amsterdam, NL

${ }^{102}$ Psychiatry, North East London NHS Foundation Trust, llford, GB

${ }^{103}$ Department of Neurodegenerative Diseases and Geropsychiatry, University Hospital Bonn, Bonn, DE

${ }^{104}$ Psychiatric and Neurodevelopmental Genetics Unit, Massachusetts General Hospital, Boston, MA, US

${ }^{105}$ HudsonAlpha Institute for Biotechnology, Huntsville, AL, US

${ }^{106}$ Department of Human Genetics, University of Michigan, Ann Arbor, MI, US

${ }^{107}$ Psychiatry, University of Illinois at Chicago College of Medicine, Chicago, IL, US

${ }^{108}$ Max Planck Institute of Psychiatry, Munich, DE

${ }^{109}$ Mental Health, NHS 24, Glasgow, GB

${ }^{110}$ Division of Psychiatry, Centre for Clinical Brain Sciences, University of Edinburgh, Edinburgh, GB

${ }^{111}$ Psychiatry, Brigham and Women's Hospital, Boston, MA, US

${ }^{112}$ Department of Psychiatry and Psychotherapy, University of Bonn, Bonn, DE

${ }^{113}$ Department of Genetics, Harvard Medical School, Boston, MA, US

${ }^{114}$ Department of Psychiatry, University of Michigan, Ann Arbor, MI, US

${ }^{115}$ Genetic Cancer Susceptibility Group, International Agency for Research on Cancer, Lyon, FR

${ }^{116}$ Estonian Genome Center, University of Tartu, Tartu, EE 
117Discipline of Biochemistry, Neuroimaging and Cognitive Genomics (NICOG)

Centre, National University of Ireland, Galway, Galway, IE

${ }^{118}$ Neuropsychiatric Genetics Research Group, Dept of Psychiatry and Trinity

Translational Medicine Institute, Trinity College Dublin, Dublin, IE

${ }^{119}$ Institute of Neuroscience and Medicine (INM-1), Research Centre Jülich, Jülich, DE

${ }^{120}$ Research/Psychiatry, Veterans Affairs San Diego Healthcare System, San Diego, CA, US

${ }^{121}$ Department of Clinical Sciences, Psychiatry, Umeå University Medical Faculty, Umeå, SE

${ }^{122}$ Department of Clinical Psychiatry, Psychiatry Clinic, Clinical Center University of Sarajevo, Sarajevo, BA

${ }^{123}$ Department of Neurobiology, Care sciences, and Society, Karolinska Institutet and Center for Molecular Medicine, Karolinska University Hospital, Stockholm, SE

${ }^{124}$ Psychiatry, Harvard Medical School, Boston, MA, US

${ }^{125}$ Division of Clinical Research, Massachusetts General Hospital, Boston, MA, US

${ }^{126}$ Outpatient Clinic for Bipolar Disorder, Altrecht, Utrecht, NL

${ }^{127}$ Department of Psychiatry, Washington University in Saint Louis, Saint Louis, MO, US

${ }^{128}$ Department of Biochemistry and Molecular Biology II, Institute of Neurosciences, Center for Biomedical Research, University of Granada, Granada, ES

${ }^{129}$ Department of Neuroscience, Icahn School of Medicine at Mount Sinai, New York, NY, US

${ }^{130}$ Medicine, Psychiatry, Biomedical Informatics, Vanderbilt University Medical Center, Nashville, TN, US

${ }^{131}$ Department of Health Sciences Research, Mayo Clinic, Rochester, MN, US

${ }^{132}$ Psychiatry and Behavioral Sciences, Stanford University School of Medicine, Stanford, CA, US

${ }^{133}$ Rush University Medical Center, Chicago, IL, US

${ }^{134}$ Scripps Translational Science Institute, La Jolla, CA, US

${ }^{135}$ Faculty of Medicine, Department of Psychiatry, School of Health Sciences, University of Iceland, Reykjavik, IS

${ }^{136}$ Div Mental Health and Addiction, Oslo University Hospital, Oslo, NO

${ }^{137}$ NORMENT, University of Oslo, Oslo, NO

${ }^{138}$ Psychiatry and the Behavioral Sciences, University of Southern California, Los Angeles, CA, US 
${ }^{139}$ Mood Disorders, PsyQ, Rotterdam, NL

${ }^{140}$ Institute for Medical Sciences, University of Aberdeen, Aberdeen, UK

${ }^{141}$ Research Division, Federal Institute for Drugs and Medical Devices (BfArM), Bonn, DE

${ }^{142}$ Centre for Addiction and Mental Health, Toronto, ON, CA

${ }^{143}$ Neurogenomics, TGen, Los Angeles, AZ, US

${ }^{144}$ Psychiatry, Psychiatrisches Zentrum Nordbaden, Wiesloch, DE

${ }^{145}$ Computational Sciences Center of Emphasis, Pfizer Global Research and Development, Cambridge, MA, US

${ }^{146}$ Department of Biostatistics, Princess Margaret Cancer Centre, Toronto, ON, CA

${ }^{147}$ Dalla Lana School of Public Health, University of Toronto, Toronto, ON, CA

${ }^{148}$ Psychological Medicine, Institute of Psychiatry, Psychology \& Neuroscience, King's College London, London, GB

${ }^{149}$ Department of Mental Health, Johns Hopkins University Bloomberg School of Public Health, Baltimore, MD, US

${ }^{150}$ Institute of Genetic Medicine, Johns Hopkins University School of Medicine, Baltimore, MD, US

${ }^{151} \mathrm{~A}$ list of members appears in the Supplementary Note

${ }^{152 N O R M E N T, ~ K G ~ J e b s e n ~ C e n t r e ~ f o r ~ P s y c h o s i s ~ R e s e a r c h, ~ D i v i s i o n ~ o f ~ M e n t a l ~}$ Health and Addiction, Institute of Clinical Medicine and Diakonhjemmet Hospital, University of Oslo, Oslo, NO

${ }^{153}$ National Institute of Mental Health, Klecany, CZ

${ }^{154}$ Department of Psychiatry, University of Melbourne, Melbourne, Vic, AU

${ }^{155}$ Department of Psychiatry, University of Münster, Münster, DE

${ }^{156}$ Department of Psychiatry and Addiction Medicine, Assistance Publique Hôpitaux de Paris, Paris, FR

${ }^{157}$ Paris Bipolar and TRD Expert Centres, FondaMental Foundation, Paris, FR

158UMR-S1144 Team 1: Biomarkers of relapse and therapeutic response in addiction and mood disorders, INSERM, Paris, FR

${ }^{159}$ Psychiatry, Université Paris Diderot, Paris, FR

${ }^{160}$ Psychiatry, University of Pennsylvania, Philadelphia, PA, US

${ }^{161}$ Division of Endocrinology, Children's Hospital Boston, Boston, MA, US

${ }^{162}$ Centre for Affective Disorders, Institute of Psychiatry, Psychology and Neuroscience, London, GB

${ }^{163}$ Department of Psychiatry \& Psychology, Mayo Clinic, Rochester, MN, US 
${ }^{164}$ School of Medical Sciences, University of New South Wales, Sydney, NSW, AU

${ }^{165}$ Department of Human Genetics, University of Chicago, Chicago, IL, US

${ }^{166}$ Biometric Psychiatric Genetics Research Unit, Alexandru Obregia Clinical Psychiatric Hospital, Bucharest, RO

${ }^{167}$ Institute of Neuroscience and Physiology, University of Gothenburg, Gothenburg, SE

${ }^{168}$ INSERM, Paris, FR

${ }^{169}$ Department of Medical \& Molecular Genetics, King's College London, London, GB

${ }^{170}$ Neuroscience Therapeutic Area, Janssen Research and Development, LLC, Titusville, NJ, US

${ }^{171}$ Cancer Epidemiology and Prevention, M. Sklodowska-Curie Cancer Center and Institute of Oncology, Warsaw, PL

${ }^{172}$ School of Psychology, The University of Queensland, Brisbane, QLD, AU

${ }^{173}$ Research Institute, Lindner Center of HOPE, Mason, $\mathrm{OH}$, US

${ }^{174}$ Centre for Cognitive Ageing and Cognitive Epidemiology, University of Edinburgh, Edinburgh, GB

${ }^{175}$ Human Genetics Branch, Intramural Research Program, National Institute of Mental Health, Bethesda, MD, US

${ }^{176}$ Division of Mental Health and Addiction, Oslo University Hospital, Oslo, NO

${ }^{177}$ Division of Mental Health and Addiction, University of Oslo, Institute of Clinical Medicine, Oslo, NO

${ }^{178}$ Institute of Molecular and Cell Biology, University of Tartu, Tartu, EE

${ }^{179}$ Mental Health, Faculty of Medicine and Health Sciences, Norwegian University of Science and Technology - NTNU, Trondheim, NO

${ }^{180}$ Psychiatry, St Olavs University Hospital, Trondheim, NO

${ }^{181}$ Psychosis Research Unit, Aarhus University Hospital, Risskov, DK

${ }^{182}$ Munich Cluster for Systems Neurology (SyNergy), Munich, DE

${ }^{183}$ University of Liverpool, Liverpool, GB

${ }^{184}$ Psychiatry and Human Genetics, University of Pittsburgh, Pittsburgh, PA, US

${ }^{185}$ Mental Health Services in the Capital Region of Denmark, Mental Health Center Copenhagen, University of Copenhagen, Copenhagen, DK

${ }^{186}$ Division of Psychiatry, Haukeland Universitetssjukehus, Bergen, NO

${ }^{187}$ Faculty of Medicine and Dentistry, University of Bergen, Bergen, NO 
${ }^{188}$ Human Genetics and Computational Biomedicine, Pfizer Global Research and Development, Groton, CT, US

${ }^{189}$ College of Medicine Institute for Genomic Health, SUNY Downstate Medical Center College of Medicine, Brooklyn, NY, US

${ }^{190}$ Department of Clinical Genetics, Amsterdam Neuroscience, Vrije Universiteit Medical Center, Amsterdam, NL

${ }^{191}$ Department of Neurology and Neurosurgery, McGill University, Faculty of Medicine, Montreal, QC, CA

${ }^{192}$ Montreal Neurological Institute and Hospital, Montreal, QC, CA

${ }^{193}$ Department of Biomedical and NeuroMotor Sciences, University of Bologna, Bologna, IT

${ }^{194}$ Department of Psychiatry, Massachusetts General Hospital, Boston, MA, US

${ }^{195}$ Psychiatric and Neurodevelopmental Genetics Unit (PNGU), Massachusetts General Hospital, Boston, MA, US

${ }^{196}$ Faculty of Medicine, University of Iceland, Reykjavik, IS

${ }^{197}$ Department of Psychiatry, Hospital Namsos, Namsos, NO

${ }^{198}$ Department of Mental Health, Norwegian University of Science and Technology, Trondheim NO

${ }^{199}$ Department of Genetics, University of North Carolina at Chapel Hill, Chapel Hill, NC, US

${ }^{200}$ Department of Psychiatry, University of North Carolina at Chapel Hill, Chapel Hill, NC, US

${ }^{201}$ Department of Psychiatry, McGill University, Montreal, QC, CA

${ }^{202}$ Dept of Psychiatry, Sankt Olavs Hospital Universitetssykehuset i Trondheim, Trondheim, NO

${ }^{203}$ Clinical Institute of Neuroscience, Hospital Clinic, University of Barcelona, IDIBAPS, CIBERSAM, Barcelona, ES

${ }^{204}$ Institute of Biological Psychiatry, MHC Sct. Hans, Mental Health Services Copenhagen, Roskilde, DK

${ }^{205}$ Department of Clinical Medicine, University of Copenhagen, Copenhagen, DK

${ }^{206}$ Psychiatry, Indiana University School of Medicine, Indianapolis, IN, US

${ }^{207}$ Biochemistry and Molecular Biology, Indiana University School of Medicine, Indianapolis, IN, US

\section{Acknowledgments:}

This paper is dedicated to the memory of Psychiatric Genomics Consortium (PGC) founding member and Bipolar disorder working group co-chair Pamela Sklar. We thank the participants who donated their time, experiences and 
DNA to this research, and to the clinical and scientific teams that worked with them. We are deeply indebted to the investigators who comprise the PGC. The views expressed are those of the authors and not necessarily those of any funding or regulatory body. Analyses were carried out on the NL Genetic Cluster Computer (http:// www.geneticcluster.org ) hosted by SURFsara, and the Mount Sinai high performance computing cluster (http:// hpc.mssm.edu). PGC members have received major funding from the US National Institute of Mental Health. This work was funded in part by the Brain and Behavior Research Foundation, Stanley Medical Research Institute, University of Michigan, Pritzker Neuropsychiatric Disorders Research Fund L.L.C., Marriot Foundation and the Mayo Clinic Center for Individualized Medicine, the NIMH Intramural Research Program; Canadian Institutes of Health Research; the UK Maudsley NHS Foundation Trust, NIHR, NRS, MRC, Wellcome Trust; European Research Council; German Ministry for Education and Research, German Research Foundation IZKF of Münster, Deutsche Forschungsgemeinschaft, ImmunoSensation, the Dr. Lisa-Oehler Foundation, University of Bonn; the Swiss National Science Foundation; French Foundation FondaMental and ANR; Spanish Ministerio de Economía, CIBERSAM, Industria y Competitividad, European Regional Development Fund (ERDF), Generalitat de Catalunya, EU Horizon 2020 Research and Innovation Programme; BBMRI-NL; South-East Norway Regional Health Authority and Mrs. Throne-Holst; Swedish Research Council, Stockholm County Council, Söderström Foundation; Lundbeck Foundation, Aarhus University; Australia NHMRC, NSW Ministry of Health, Janette M O'Neil and Betty C Lynch. The views expressed are those of the authors and not necessarily those of their institutions or funding bodies. Additional acknowledgements and including funding sources are presented in the Supplementary Note.

\section{References:}

1. Ferrari AJ et al. The prevalence and burden of bipolar disorder: findings from the Global Burden of Disease Study 2013. Bipolar Disord. 18, 440-450 (2016). [PubMed: 27566286]

2. Lichtenstein $P$ et al. Common genetic determinants of schizophrenia and bipolar disorder in Swedish families: a population-based study. Lancet 373, 234-239 (2009). [PubMed: 19150704]

3. Edvardsen J et al. Heritability of bipolar spectrum disorders. Unity or heterogeneity? J. Affect. Disord 106, 229-240 (2008). [PubMed: 17692389]

4. McGuffin $P$ et al. The heritability of bipolar affective disorder and the genetic relationship to unipolar depression. Arch. Gen. Psychiatry 60, 497-502 (2003). [PubMed: 12742871]

5. American Psychiatric Association. Diagnostic and Statistical Manual of Mental Disorders (DSM-5®). (American Psychiatric Pub, 2013).

6. World Health Organization. The ICD-10 Classification of Mental and Behavioural Disorders: Clinical Descriptions and Diagnostic Guidelines. (World Health Organization, 1992).

7. Craddock N \& Owen MJ The Kraepelinian dichotomy - going, going... but still not gone. Br. J. Psychiatry 196, 92-95 (2010). [PubMed: 20118450]

8. Lee $\mathrm{SH}$ et al. Genetic relationship between five psychiatric disorders estimated from genome-wide SNPs. Nat. Genet 45, 984-994 (2013). [PubMed: 23933821]

9. Sklar P et al. Large-scale genome-wide association analysis of bipolar disorder identifies a new susceptibility locus near ODZ4. Nat. Genet 43, 977-U162 (2011). [PubMed: 21926972]

10. Baum AE et al. A genome-wide association study implicates diacylglycerol kinase eta (DGKH) and several other genes in the etiology of bipolar disorder. Mol. Psychiatry 13, 197-207 (2008). [PubMed: 17486107]

11. Charney AW et al. Evidence for genetic heterogeneity between clinical subtypes of bipolar disorder. Transl. Psychiatry 7, e993 (2017). [PubMed: 28072414]

12. Chen DT et al. Genome-wide association study meta-analysis of European and Asian-ancestry samples identifies three novel loci associated with bipolar disorder. Mol. Psychiatry 18, 195-205 (2013). [PubMed: 22182935]

13. Cichon $\mathrm{S}$ et al. Genome-wide association study identifies genetic variation in neurocan as a susceptibility factor for bipolar disorder. Am. J. Hum. Genet 88, 372-381 (2011). [PubMed: 21353194]

14. Ferreira MAR et al. Collaborative genome-wide association analysis supports a role for ANK3 and CACNA1C in bipolar disorder. Nat. Genet 40, 1056-1058 (2008). [PubMed: 18711365]

15. Green EK et al. Association at SYNE1 in both bipolar disorder and recurrent major depression. Mol. Psychiatry 18, 614-617 (2013). [PubMed: 22565781] 
16. Green EK et al. Replication of bipolar disorder susceptibility alleles and identification of two novel genome-wide significant associations in a new bipolar disorder case-control sample. Mol. Psychiatry 18, 1302-1307 (2013). [PubMed: 23070075]

17. Hou L et al. Genome-wide association study of 40,000 individuals identifies two novel loci associated with bipolar disorder. Hum. Mol. Genet 25, 3383-3394 (2016). [PubMed: 27329760]

18. Mühleisen TW et al. Genome-wide association study reveals two new risk loci for bipolar disorder. Nat. Commun 5, 3339 (2014). [PubMed: 24618891]

19. Schulze TG et al. Two variants in Ankyrin 3 (ANK3) are independent genetic risk factors for bipolar disorder. Mol. Psychiatry 14, 487-491 (2009). [PubMed: 19088739]

20. Scott LJ et al. Genome-wide association and meta-analysis of bipolar disorder in individuals of European ancestry. Proc. Natl. Acad. Sci. U. S. A 106, 7501-7506 (2009). [PubMed: 19416921]

21. Sklar P et al. Whole-genome association study of bipolar disorder. Mol. Psychiatry 13, 558-569 (2008). [PubMed: 18317468]

22. Smith EN et al. Genome-wide association study of bipolar disorder in European American and African American individuals. Mol. Psychiatry 14, 755-763 (2009). [PubMed: 19488044]

23. Burton PR et al. Genome-wide association study of 14,000 cases of seven common diseases and 3,000 shared controls. Nature 447, 661-678 (2007). [PubMed: 17554300]

24. Gratten J, Wray NR, Keller MC \& Visscher PM Large-scale genomics unveils the genetic architecture of psychiatric disorders. Nat. Neurosci 17, 782-790 (2014). [PubMed: 24866044]

25. Bulik-Sullivan BK et al. LD Score regression distinguishes confounding from polygenicity in genome-wide association studies. Nat. Genet 47, 291-295 (2015). [PubMed: 25642630]

26. Zheng J et al. LD Hub: a centralized database and web interface to perform LD score regression that maximizes the potential of summary level GWAS data for SNP heritability and genetic correlation analysis. Bioinformatics 33, 272-279 (2017). [PubMed: 27663502]

27. Palmer C \& Pe'er I Statistical correction of the Winner's Curse explains replication variability in quantitative trait genome-wide association studies. PLoS Genet. 13, e1006916 (2017). [PubMed: 28715421]

28. Zhong H \& Prentice RL Bias-reduced estimators and confidence intervals for odds ratios in genome-wide association studies. Biostatistics 9, 621-634 (2008). [PubMed: 18310059]

29. Holland D et al. Beyond SNP Heritability: Polygenicity and Discoverability Estimated for Multiple Phenotypes with a Univariate Gaussian Mixture Model. bioRxiv 133132 (2018). doi: $10.1101 / 133132$

30. Gao X, Starmer J \& Martin ER A multiple testing correction method for genetic association studies using correlated single nucleotide polymorphisms. Genet. Epidemiol 32, 361-369 (2008). [PubMed: 18271029]

31. Schizophrenia Working Group of the Psychiatric Genomics Consortium. Biological insights from 108 schizophrenia-associated genetic loci. Nature 511, 421-427 (2014). [PubMed: 25056061]

32. Ripke $\mathrm{S}$ et al. Genome-wide association analysis identifies 13 new risk loci for schizophrenia. Nat. Genet 45, 1150-1159 (2013). [PubMed: 23974872]

33. Wray NR \& Sullivan PF Genome-wide association analyses identify 44 risk variants and refine the genetic architecture of major depression. bioRxiv (2017).

34. Okbay A et al. Genetic variants associated with subjective well-being, depressive symptoms, and neuroticism identified through genome-wide analyses. Nat. Genet (2016). doi:10.1038/ng.3552

35. Cross-Disorder Group of the Psychiatric Genomics Consortium et al. Genetic relationship between five psychiatric disorders estimated from genome-wide SNPs. Nat. Genet 45, 984-994 (2013). [PubMed: 23933821]

36. Duncan L et al. Significant Locus and Metabolic Genetic Correlations Revealed in Genome-Wide Association Study of Anorexia Nervosa. Am. J. Psychiatry appiajp201716121402 (2017).

37. Otowa $\mathrm{T}$ et al. Meta-analysis of genome-wide association studies of anxiety disorders. Mol. Psychiatry 21, 1391-1399 (2016). [PubMed: 26754954]

38. Gale $\mathrm{CR}$ et al. Pleiotropy between neuroticism and physical and mental health: findings from 108 038 men and women in UK Biobank. Transl. Psychiatry 6, e791 (2016). [PubMed: 27115122] 
39. Rietveld CA et al. GWAS of 126,559 individuals identifies genetic variants associated with educational attainment. Science 340, 1467-1471 (2013). [PubMed: 23722424]

40. Okbay A et al. Genome-wide association study identifies 74 loci associated with educational attainment. Nature 533, 539-542 (2016). [PubMed: 27225129]

41. Benyamin B et al. Childhood intelligence is heritable, highly polygenic and associated with FNBP1L. Mol. Psychiatry 19, 253-258 (2014). [PubMed: 23358156]

42. Sniekers $\mathrm{S}$ et al. Genome-wide association meta-analysis of 78,308 individuals identifies new loci and genes influencing human intelligence. Nat. Genet 49, 1107-1112 (2017). [PubMed: 28530673]

43. MacArthur $\mathrm{J}$ et al. The new NHGRI-EBI Catalog of published genome-wide association studies (GWAS Catalog). Nucleic Acids Res. 45, D896-D901 (2017). [PubMed: 27899670]

44. Hou L et al. Genome-wide association study of 40,000 individuals identifies two novel loci associated with bipolar disorder. Hum. Mol. Genet 25, 3383-3394 (2016). [PubMed: 27329760]

45. Ikeda $\mathrm{M}$ et al. A genome-wide association study identifies two novel susceptibility loci and trans population polygenicity associated with bipolar disorder. Mol. Psychiatry (2017). doi:10.1038/mp. 2016.259

46. Finucane HK et al. Partitioning heritability by functional annotation using genome-wide association summary statistics. Nat. Genet 47, 1228-1235 (2015). [PubMed: 26414678]

47. Pers TH et al. Biological interpretation of genome-wide association studies using predicted gene functions. Nat. Commun 6, 5890 (2015). [PubMed: 25597830]

48. Zhu Z et al. Integration of summary data from GWAS and eQTL studies predicts complex trait gene targets. Nat. Genet 48, 481-487 (2016). [PubMed: 27019110]

49. Pavlides JMW et al. Predicting gene targets from integrative analyses of summary data from GWAS and eQTL studies for 28 human complex traits. Genome Med. 8, 84 (2016). [PubMed: 27506385]

50. Qi T et al. Identifying gene targets for brain-related traits using transcriptomic and methylomic data from blood. Nat. Commun 9, 2282 (2018). [PubMed: 29891976]

51. de Leeuw CA, Mooij JM, Heskes T \& Posthuma D MAGMA: generalized gene-set analysis of GWAS data. PLoS Comput. Biol 11, e1004219 (2015). [PubMed: 25885710]

52. Bipolar Disorder and Schizophrenia Working Group of the Psychiatric Genomics Consortium. Genomic Dissection of Bipolar Disorder and Schizophrenia, Including 28 Subphenotypes. Cell 173, 1705-1715.e16 (2018). [PubMed: 29906448]

53. Lee SH, Goddard ME, Wray NR \& Visscher PM A better coefficient of determination for genetic profile analysis. Genet. Epidemiol 36, 214-224 (2012). [PubMed: 22714935]

54. Simonsen $\mathrm{C}$ et al. Neurocognitive dysfunction in bipolar and schizophrenia spectrum disorders depends on history of psychosis rather than diagnostic group. Schizophr. Bull 37, 73-83 (2011). [PubMed: 19443616]

55. Goes FS et al. Genome-wide association of mood-incongruent psychotic bipolar disorder. Transl. Psychiatry 2, e180 (2012). [PubMed: 23092984]

56. Allardyce J et al. Association Between Schizophrenia-Related Polygenic Liability and the Occurrence and Level of Mood-Incongruent Psychotic Symptoms in Bipolar Disorder. JAMA Psychiatry 75, 28-35 (2018). [PubMed: 29167880]

57. Palmer C \& Pe'er I Statistical Correction of the Winner9s Curse Explains Replication Variability in Quantitative Trait Genome-Wide Association Studies. bioRxiv 104786 (2017).

58. Goes FS et al. Genome-wide association study of schizophrenia in Ashkenazi Jews. Am. J. Med. Genet. B Neuropsychiatr. Genet 168, 649-659 (2015). [PubMed: 26198764]

59. Gaspar HA \& Breen G Pathways analyses of schizophrenia GWAS focusing on known and novel drug targets. Biorxiv (2017). doi:10.1101/091264

60. Camandola S \& Mattson MP Aberrant subcellular neuronal calcium regulation in aging and Alzheimer's disease. Biochimica et Biophysica Acta (BBA) - Molecular Cell Research 1813, 965973 (2011). [PubMed: 20950656]

61. Mertens J et al. Differential responses to lithium in hyperexcitable neurons from patients with bipolar disorder. Nature 527, 95-99 (2015). [PubMed: 26524527] 
62. Yang C-P et al. Comprehensive integrative analyses identify GLT8D1 and CSNK2B as schizophrenia risk genes. Nat. Commun 9, 838 (2018). [PubMed: 29483533]

63. Lee S-H, Zabolotny JM, Huang H, Lee H \& Kim Y-B Insulin in the nervous system and the mind: Functions in metabolism, memory, and mood. Mol Metab 5, 589-601 (2016). [PubMed: 27656397]

64. McIntyre RS et al. A randomized, double-blind, controlled trial evaluating the effect of intranasal insulin on neurocognitive function in euthymic patients with bipolar disorder. Bipolar Disord. 14, 697-706 (2012). [PubMed: 23107220]

65. Desfossés J, Stip E, Bentaleb LA \& Potvin S Endocannabinoids and Schizophrenia. Pharmaceuticals 3, 3101-3126 (2010).

66. Zamberletti E, Rubino T \& Parolaro D The endocannabinoid system and schizophrenia: integration of evidence. Curr. Pharm. Des 18, 4980-4990 (2012). [PubMed: 22716159]

67. Serra G \& Fratta W A possible role for the endocannabinoid system in the neurobiology of depression. Clin. Pract. Epidemiol. Ment. Health 3, 25 (2007). [PubMed: 18021439]

68. MacCabe JH et al. Excellent school performance at age 16 and risk of adult bipolar disorder: national cohort study. Br. J. Psychiatry 196, 109-115 (2010). [PubMed: 20118454]

69. Vreeker A et al. High educational performance is a distinctive feature of bipolar disorder: a study on cognition in bipolar disorder, schizophrenia patients, relatives and controls. Psychol. Med 46, 807-818 (2016). [PubMed: 26621616]

\section{Methods-only References:}

70. Ripke S Ricopili: a tool for visualizing regions of interest in select GWAS data sets. (2014).

71. Purcell S et al. PLINK: a tool set for whole-genome association and population-based linkage analyses. Am. J. Hum. Genet 81, 559-575 (2007). [PubMed: 17701901]

72. Chang $\mathrm{CC}$ et al. Second-generation PLINK: rising to the challenge of larger and richer datasets. Gigascience 4, 7 (2015). [PubMed: 25722852]

73. Euesden J, Lewis CM \& O’Reilly PF PRSice: Polygenic Risk Score software. Bioinformatics 31, 1466-1468 (2015). [PubMed: 25550326]

74. Finucane $\mathrm{H}$ et al. Heritability enrichment of specifically expressed genes identifies disease-relevant tissues and cell types. doi:10.1101/103069

75. O'Dushlaine C et al. Psychiatric genome-wide association study analyses implicate neuronal, immune and histone pathways. Nat. Neurosci 18, 199-209 (2015). [PubMed: 25599223]

76. 1000 Genomes Project Consortium et al. A global reference for human genetic variation. Nature 526, 68-74 (2015). [PubMed: 26432245]

77. Olier I, Vellido A \& Giraldo J Kernel generative topographic mapping. in ESANN 2010, 481-486 (2010). 


\section{Editorial Summary:}

Genome-wide analysis identifies 30 loci associated with bipolar disorder, allowing for comparisons of shared genes and pathways with other psychiatric disorders, including schizophrenia and depression. 


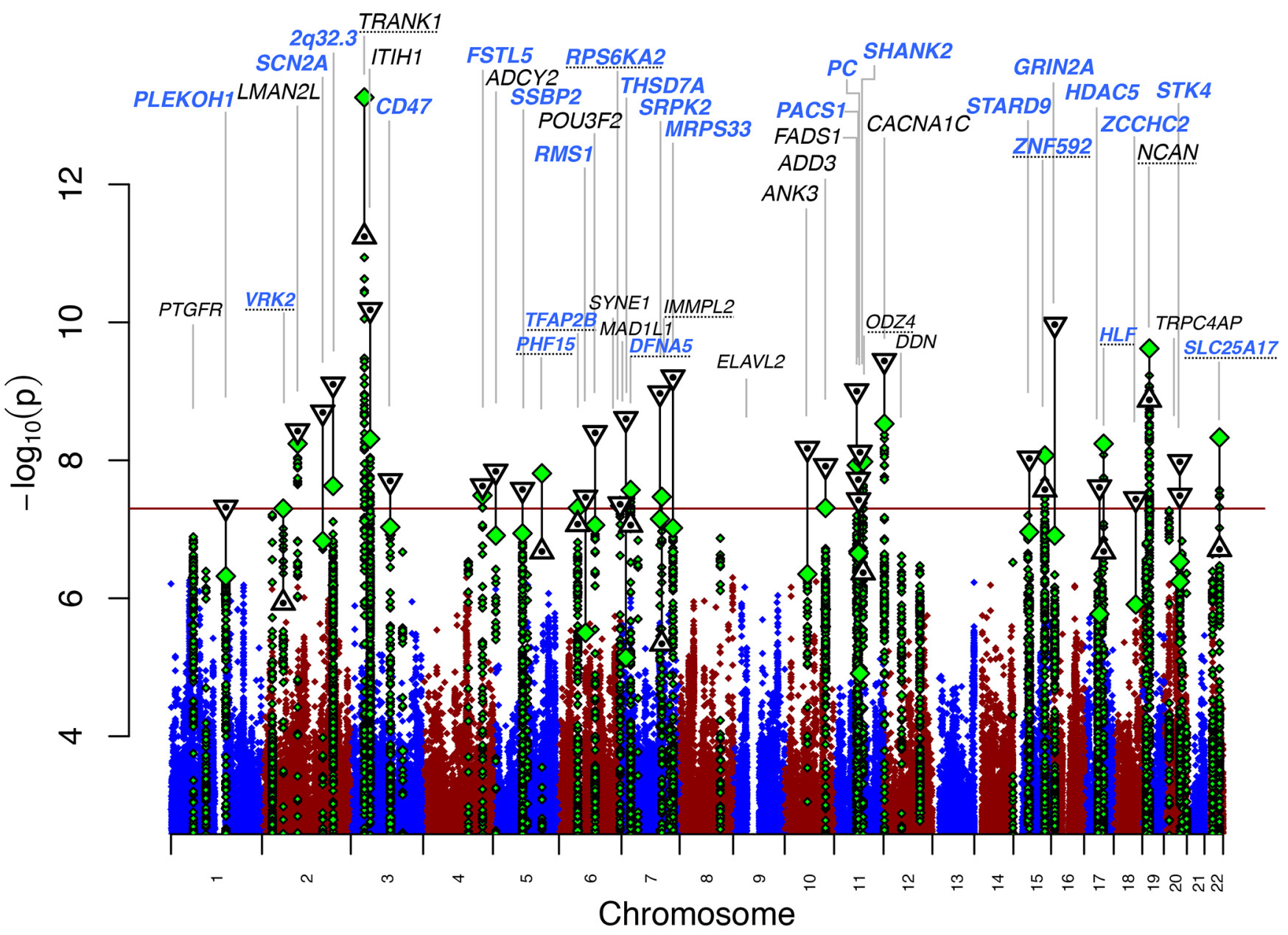

Figure 1.

Manhattan plot for our primary genomewide association analysis of 20,352 cases and 31,358 controls. GWAS $-\log _{10} \mathrm{P}$-values are plotted for all SNPs across chromosomes 1-22 (diamonds, green for loci with lead SNP GWAS $\mathrm{P}<10^{-6}$ ). Combined GWAS+followup $-\log _{10} \mathrm{P}$-values for lead SNPs reaching genome-wide significance in either GWAS or combined analysis (triangles, inverted if GWAS+followup $-\log _{10} \mathrm{P}>$ GWAS $-\log _{10} \mathrm{P}$ ). Labels correspond to gene symbols previously reported for published loci (black) and the nearest genes for novel loci (blue), at top if GWAS+followup $\mathrm{P}<5 \times 10^{-8}$. Loci with onetailed follow-up p $>0.05$ (Table 1 ) have dotted underlined locus names. 


\section{Scaled Polygenic Risk Score}

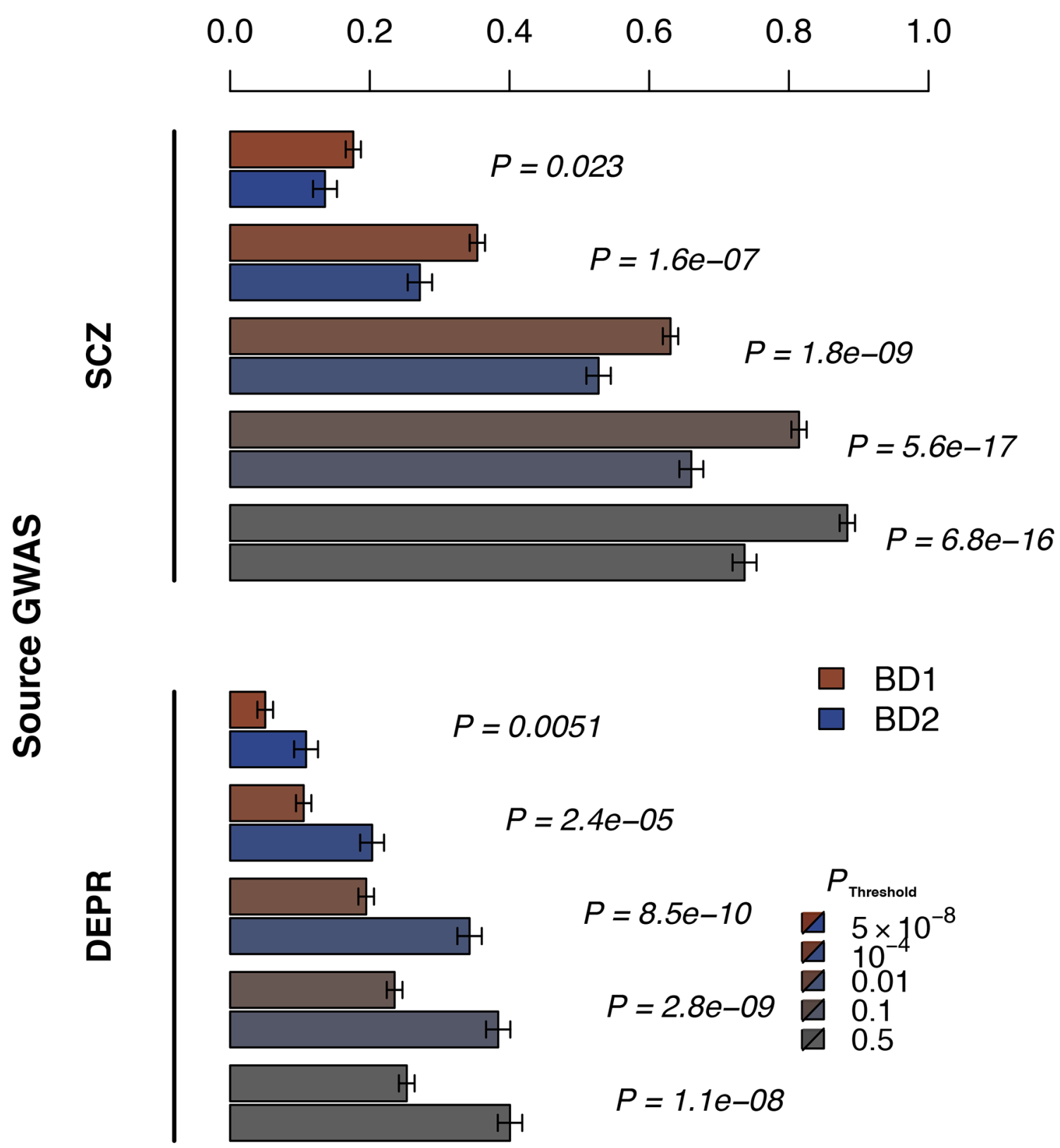

Figure 2.

Association of BD1 and BD2 subtypes with schizophrenia (SCZ) and major depression (DEPR) polygenic risk scores (PRS). Shown are mean PRS values (1 s.e. error bars), adjusted for study and ancestry covariates and scaled to the PRS mean and sd in control subjects, in BD1 (red) and BD2 (blue) cases, for increasing source GWAS P-value thresholds (increasing grey) as indicated. P-values (italics) test BD1 vs BD2 mean PRS, in 
logistic regression of case subtype on PRS with covariates. Results are detailed in Supplementary Table 13. 


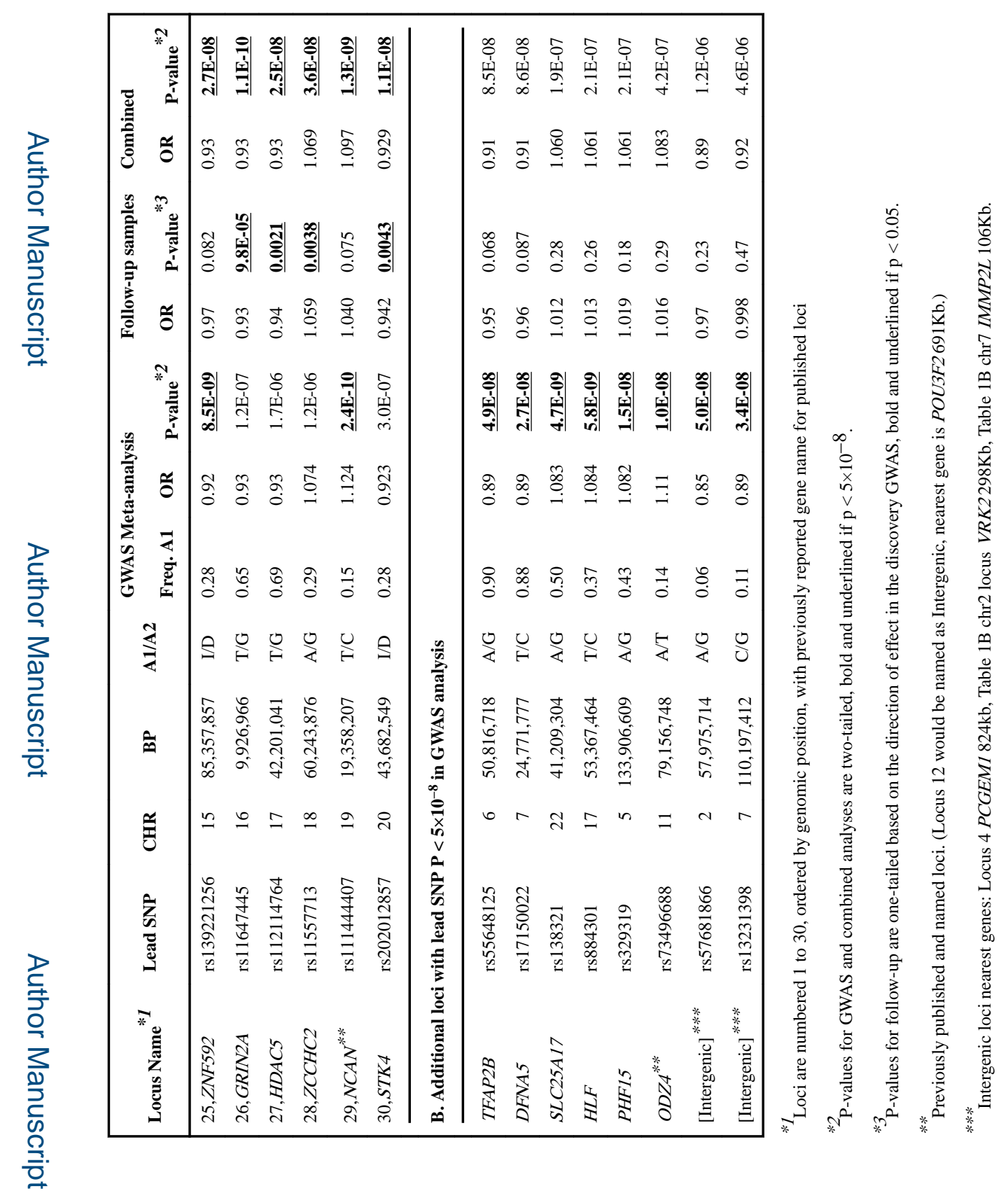

로을 